\title{
Stability properties of periodic traveling waves for the intermediate long wave equation
}

\author{
Jaime Angulo, Eleomar Cardoso Jr. and Fábio Natali
}

\begin{abstract}
In this paper we determine orbital and linear stability of a class of spatially periodic wavetrain solutions with the mean zero property related to the intermediate long wave equation. Our arguments follow recent developments for the study of the stability of periodic traveling waves.
\end{abstract}

\section{Introduction}

One of the most fascinating phenomena appearing in nonlinear dispersive equations is the existence of solutions that maintain their shape and travel with constant speed. Such solutions are the result of a perfect balance between the nonlinear and dispersive effects at the medium. In general, these solutions are called traveling waves, and it is well known that their existence has very wide applications in fluid dynamics, nonlinear optics, hydrodynamic and many other fields (see pioneering works due to Boussinesq, Benjamin-Ono, Benjamin-Bona-Mahoney, Miura, Gardner, and Kruskal). The study of the dynamics of these solutions has become one of the important issues of the last decades for evolutive nonlinear partial differential equations.

We can say that the initial impetus for the scientific activity of these profiles was the inverse scattering theory (IST) for the Korteweg-de Vries equation (KdVequation henceforth)

$$
u_{t}+u_{x}+\left(u^{2}\right)_{x}+u_{x x x}=0 .
$$

One of the lessons learned from the IST is that the traveling wave with a solitary wave profile, namely, $u(x, t)=\psi(x-c t)$ with $c>0$ and

$$
\lim _{|\xi| \rightarrow+\infty} \psi(\xi)=0
$$

plays a central role in the long-time asymptotics of solutions to the initial-value problem associated to the KdV-equation. Indeed, general classes of initial data are 
known that evolve as a finite sequence of solitary waves followed by a dispersive tail. A companion result is that individual solitary waves are orbitally stable solutions of the evolution equation. The exact theory of stability of solitary waves for the KdV-equation was started by Benjamin in [12] (see also Bona in [14]), and reached maturity a decade ago with works due to Albert [4], Albert and Bona [5], Albert, Bona and Henry [6] and Weinstein [38], [40]. Next, in papers due to Strauss et al. and Weinstein [15], [24], [39] it was shown that not all solitary wave solutions are stable. Both necessary and sufficient conditions for stability of the traveling waves solutions of a range of nonlinear dispersive evolution equations appear in various of the above references.

In the last years, the study of stability of traveling waves of periodic type associated with nonlinear dispersive equations has increased significantly. A rich variety of new mathematical problems have emerged, as well as the physical importance related to them. This subject is often studied in relation to the natural symmetries associated to the model (translation invariance and/or rotation invariance) and by perturbations of symmetric classes, e.g., the class of periodic functions with the same minimal period as the underlying wave. In the case of shallow-water wave models (or long internal waves in a density-stratified ocean, ion-acoustic waves in a plasma or acoustic waves on a crystal lattice), a formal stability theory of periodic traveling wave has started with the pioneering work of Benjamin [13] regarding the periodic steady solutions called cnoidal waves for the KdV equation. The waveform profiles were found first by Korteweg and de Vries for the KdV-equation. The cnoidal traveling wave solution, namely, $u(x, t)=\varphi_{c}(x-c t)$, has a profile given by

$$
\varphi_{c}(\xi)=\beta_{2}+\left(\beta_{3}-\beta_{2}\right) \mathrm{cn}^{2}\left(\sqrt{\frac{\beta_{3}-\beta_{1}}{12}} \xi ; k\right),
$$

where $\operatorname{cn}(\cdot ; k)$ represents the Jacobi elliptic function called cnoidal associated with the elliptic modulus $k \in(0,1)$, and the $\beta_{i}$ 's are real constants satisfying the classical relations

$$
\beta_{1}<\beta_{2}<\beta_{3}, \quad \beta_{1}+\beta_{2}+\beta_{3}=3 c, \quad k^{2}=\frac{\beta_{3}-\beta_{2}}{\beta_{3}-\beta_{1}} .
$$

We recall that $\varphi_{c}$ satisfies the second order differential equation

$$
-\varphi_{c}^{\prime \prime}(\xi)+c \varphi_{c}(\xi)-\frac{1}{2} \varphi_{c}^{2}(\xi)=A_{\varphi_{c}}, \quad \xi \in \mathbb{R}
$$

with $A_{\varphi_{c}}=-\frac{1}{6} \sum_{i<j} \beta_{i} \beta_{j}$, and that the formula (1.1) is deduced from the theory of elliptic integrals and elliptic functions (see Angulo [8]). The existence of a smooth curve of solutions for (1.3) with a minimal period $L, c \in I \subset \mathbb{R} \rightarrow \varphi_{c} \in H_{\text {per }}^{n}([0, L])$ follows from the implicit function theorem. The interval $I$ in general depends of the qualitative properties of $\varphi_{c}$. In fact, if $\varphi_{c}$ has the mean zero property,

$$
\int_{0}^{L} \varphi_{c}(\xi) d \xi=0
$$


we have $I=(0,+\infty)$. If $A_{\varphi_{c}}=0$ and $\varphi_{c}(\xi)>0$, for all $\xi \in \mathbb{R}$, we have $I=$ $\left(4 \pi^{2} / L^{2},+\infty\right)$. A first stability approach for the cnoidal wave profile (1.1) was introduced by Benjamin in [13] regarding the stability in $H_{\text {per }}^{1}([0, L])$ of the orbit

$$
\Omega_{\varphi_{c}}=\left\{\varphi_{c}(\cdot+y): y \in \mathbb{R}\right\},
$$

by the periodic flow of the KdV equation. Years later, a complete study was carried out by Angulo, Bona and Scialom in [9] (see also [8]).

Recently, Angulo and Natali in [10] (see also [8]) have established a new approach for studying the stability of even and positive periodic traveling waves solutions associated to the general dispersive model

$$
u_{t}+2 u u_{x}-(\mathcal{M} u)_{x}=0,
$$

where $\mathcal{M}$ is a differential or pseudo-differential operator in the framework of periodic functions. $\mathcal{M}$ is defined as a Fourier multiplier operator by

$$
\widehat{\mathcal{M} g}(n)=\theta(n) \widehat{g}(n), \quad n \in \mathbb{Z},
$$

where the symbol $\theta$ of $\mathcal{M}$ is assumed to be a measurable, locally bounded function on $\mathbb{R}$, satisfying the condition

$$
a_{1}|n|^{m_{1}} \leq \theta(n) \leq a_{2}(1+|n|)^{m_{2}},
$$

where $m_{1} \leq m_{2},|n| \geqq n_{0}, \theta(n)>b$ for all $n \in \mathbb{Z}$, and $a_{i} \geq 0$. One of the advantages of Angulo and Natali approach is the possibility of studying non-local evolution models in a periodic framework. For instance, let us consider the case of the Benjamin-Ono equation (henceforth BO-equation)

$$
u_{t}+u u_{x}-\mathcal{H} u_{x x}=0,
$$

where $\mathcal{H}$ denotes the periodic Hilbert transform, defined for $L$-periodic functions $f$ as

$$
\mathcal{H} f(x)=\frac{1}{L} \text { p.v. } \int_{-L / 2}^{L / 2} \cot \left[\frac{\pi(x-y)}{L}\right] f(y) d y,
$$

where p.v. represents the Cauchy principal value of the integral. The Fourier transform of $\mathcal{H} f$ is given by the sequence $\{\widehat{\mathcal{H} f}(n)\}_{n \in \mathbb{Z}}$, where $\widehat{\mathcal{H} f}(n)=-i \operatorname{sgn}(n) \widehat{f}(n)$. In other words, we have that $\mathcal{M}=\mathcal{H} \partial_{x}$ has a symbol given by $\theta(n)=|n|$. The periodic traveling waves $u(x, t)=\varphi_{c}(x-c t)$ for the BO-equation with minimal period $L$ satisfy the following non-local pseudo-differential equation:

$$
\mathcal{H} \varphi_{c}+c \varphi_{c}-\frac{1}{2} \varphi_{c}^{2}=0
$$

and they are given by

$$
\varphi_{c}(x)=\frac{4 \pi}{L} \frac{\sinh (\gamma)}{\cosh (\gamma)-\cos (2 \pi x / L)},
$$

where $\gamma>0$ satisfies $\tanh (\gamma)=2 \pi /(c L)$ (therefore the wave speed $c$ must satisfy $c>2 \pi / L)$. In [10], the authors showed the first orbital stability result for the orbit generated by $\varphi_{c}$. 
In this paper, we are interested in studying the orbital and linear stability of a periodic family of traveling waves for the physically relevant intermediate long wave equation (ILW-equation henceforth),

$$
u_{t}+2 u u_{x}+\delta^{-1} u_{x}-\left(\mathcal{T}_{\delta} u\right)_{x x}=0, \quad \delta>0,
$$

with $u=u(x, t)$ a $L$-periodic function and $x, t \in \mathbb{R}$. The linear operator $\mathcal{T}_{\delta}$ is defined by

$$
\mathcal{T}_{\delta} u(x)=\frac{1}{L} \mathrm{p} \cdot \mathrm{v} \cdot \int_{-L / 2}^{L / 2} \Gamma_{\delta, L}(x-y) u(y) d y,
$$

where

$$
\Gamma_{\delta, L}(\xi)=-i \sum_{n \neq 0} \operatorname{coth}\left(\frac{2 \pi n \delta}{L}\right) e^{2 i n \pi \xi / L} .
$$

Thus, via the Fourier transform we obtain that

$$
\mathcal{T}_{\delta} u(x)=-i \sum_{n \neq 0} \operatorname{coth}\left(\frac{2 \pi n \delta}{L}\right) \widehat{u}(n) e^{2 i n \pi \xi / L} .
$$

Moreover, for $\delta \rightarrow \infty, L$ fixed, we have (see [2])

$$
\lim _{\delta \rightarrow \infty} \Gamma_{\delta, L}(x)=-\cot \left(\frac{\pi x}{L}\right)
$$

which is the kernel of the Hilbert transform in (1.9). Therefore, the ILW equation (1.10) is the natural periodic extension of the BO-equation (1.8). We note that the ILW equation is an example of the class of dispersive models (1.5) with $\mathcal{M}_{\delta}=\mathcal{T}_{\delta} \partial_{x}-1 / \delta$

One of the main objectives in this paper will be to find periodic solutions for (1.10) of the form $u(x, t)=\varphi_{c}(x-c t)$ with the periodic profile $\varphi_{c}$ having mean zero and satisfying

$$
-c \varphi_{c}+\varphi_{c}^{2}-\mathcal{M}_{\delta} \varphi_{c}=A_{c}
$$

where $A_{c}$ will be an integration constant given by $A_{c}=\frac{1}{L} \int_{0}^{L} \varphi_{c}^{2}(x) d x$. In section 3 , we obtain the following property associated to the pseudo-differential equation (1.11):

$(P 0)$ There is a smooth curve of even periodic solutions for (1.11) with the mean zero property, in the form

$$
c \in I \subset \mathbb{R} \mapsto \varphi_{c} \in H_{\text {per }}^{n}([0, L]), \quad n \in \mathbb{N},
$$

all of them with the same minimal period $L>0$.

By following arguments of Parker [37] (see also Nakamura and Matsuno in [36]), we obtain the following formula for even periodic solution for (1.11) with the mean zero property (see section 3 below):

$$
\varphi_{c}(x)=\frac{2 K(k) i}{L}\left[Z\left(\frac{2 K(k)}{L}(x-i \delta) ; k\right)-Z\left(\frac{2 K(k)}{L}(x+i \delta) ; k\right)\right],
$$


where $K(k)$ denotes the complete elliptic integral of the first kind, $Z$ is the Jacobi zeta function and $k \in(0,1)$ (see notation section below). For fixed $L$ and $\delta$, the wave speed $c$ and the elliptic modulus $k$ must satisfy specific restrictions.

Another issue of our study will be the stability of the periodic profile $\varphi_{c}$. There are two common approaches to the stability question. Firstly, we can analyze the nonlinear initial-value problem governing the difference between an arbitrary solution of the ILW equation and a given exact solution representing a wavetrain, the profile $\varphi_{c}$. In the first approximation, we assume that the difference is small and we linearize the evolution equation. The resulting linear equation can be studied in an appropriate frame of reference by a spectral approach. To our knowledge, the linearized spectral approach has never been established for the ILW equation. A second approach to stability is the orbital stability, more precisely, we study the Lyapunov stability property of the orbit

$$
\Omega_{\varphi_{c}}=\left\{\varphi_{c}(\cdot+y): y \in \mathbb{R}\right\}
$$

generated by the profile $\varphi_{c}$. The study of the dynamics of the set $\Omega_{\varphi_{c}}$ consist in verifying that for any initial condition $u_{0}$ close to $\Omega_{\varphi_{c}}$ we have that the solution $u(t)$ of (1.10) with $u(0)=u_{0}$ remains close to $\Omega_{\varphi_{c}}$ for all values of $t \in \mathbb{R}$. The specific notion of "close" is based in terms of the following pseudo-metric defined on a determined space $W$ : for $f, g \in W$,

$$
d_{2}(f, g)=\inf _{r \in \mathbb{R}}\left\|f-\tau_{r} g\right\|_{W},
$$

with $\tau_{r} h(x)=h(x+r)$. The translation symmetry $\tau$ enables us to form a quotient space, $W / \tau$, by identifying the translations $\tau f$ of each $f \in W$. If we consider $f$ and $g$ as elements of $W / \tau$, we obtain that $d_{2}$ represents a well-defined metric on this set. Note that in $W / \tau$, the difference $u-\varphi_{c}$ between $\varphi_{c}$ and the perturbed solution $u$ will represent the most crucial difference between two wave forms, namely, the shape. Again, according to our best knowledge, the orbital stability property associated to the profile $\varphi_{c}$ in (1.12) has never been established for the ILW equation in a periodic setting.

Next, we shall give a brief explanation of our work. Let us consider the new variable

$$
v(x, t)=u(x+c t, t)-\varphi_{c}(x),
$$

where $u$ solves (1.10) and $\varphi_{c}$ solves (1.11). Substituting this in equation (1.10) and by using (1.11), one finds that $v$ satisfies the nonlinear equation

$$
v_{t}+2 v v_{x}+2\left(v \varphi_{c}\right)_{x}-c v_{x}-\mathcal{M}_{\delta} v_{x}=0 .
$$

As a leading approximation for small perturbations, we replace (1.15) by its linearization around $\varphi_{c}$, and hence we obtain the linear equation

$$
v_{t}=\partial_{x}\left(\mathcal{M}_{\delta} v+c v-2 v \varphi_{c}\right) .
$$

Since $\varphi_{c}$ depends only on $x$, the equation (1.16) admits treatment by separation of variables, which leads naturally to a spectral problem. Then, by seeking particular 
solutions of (1.16) of the form $v(x, t)=e^{\lambda t} \psi(x)$, where $\lambda \in \mathbb{C}$, we see that $\psi$ satisfies the eigenvalue problem

$$
\partial_{x} \mathcal{L} \psi=\lambda \psi
$$

Here, $\mathcal{L}:=\mathcal{L}_{c, \delta}$ denotes the self-adjoint operator

$$
\mathcal{L}_{c, \delta}:=\mathcal{M}_{\delta}+c-2 \varphi_{c}
$$

We recall that the complex growth rate $\lambda$ appears as (spectral) parameter. Equation (1.18) will only have a nonzero solution $\psi$ in a given Banach space $Y$ for certain $\lambda \in \mathbb{C}$. A necessary condition for the stability of $\varphi_{c}$ is that there are not points $\lambda$ with $\operatorname{Re}(\lambda)>0$ (which would imply the existence of a solution $v$ of $(1.16)$ that lies in $Y$ as a function of $x$ and grows exponentially in time). If we denote by $\sigma$ the spectrum of $\partial_{x} \mathcal{L}$, the latter discussion suggests the utility of the following definition.

Definition 1.1 (Spectral stability and instability). A periodic traveling wave solution $\varphi_{c}$ of the ILW equation (1.10) is said to be spectrally stable if $\sigma \subset i \mathbb{R}$. Otherwise (i.e., if $\sigma$ contains point with $\operatorname{Re}(\lambda)>0), \varphi_{c}$ is spectrally unstable.

Since (1.16) is a real Hamiltonian equation, the spectrum $\sigma$ has some elementary symmetries; more precisely, $\sigma$ is symmetric with respect to reflection in the real and imaginary axes. Therefore, this implies that exponentially growing perturbation are always paired with exponentially decaying ones. More precisely, if we find a value of $\lambda$ such that $\operatorname{Re}(\lambda)<0$, one has a reflected $\widetilde{\lambda}$ such that $\operatorname{Re}(\widetilde{\lambda})>0$.

A spectral problem similar to (1.17) has been the focus of many research studies recently. For instance, if we restrict initially to traveling wave solutions of solitary wave type, sufficient conditions in order to get the linear stability/instability has been established for many specific dispersive equations in Kapitula and Stefanov [32]. In particular, the linear stability related to the generalized Korteweg-de Vries equation

$$
u_{t}+(p+1) u^{p} u_{x}+u_{x x x}=0, \quad p \in \mathbb{N},
$$

was obtained by using a Krein-Hamiltonian instability index to count the number of negative eigenvalues with positive real part. In the case of linear instability, Lin in [34] and Lopes in [35] have presented sufficient conditions for general dispersive models.

In a periodic framework, a general spectral problem of the form

$$
J \mathcal{L} \psi=\lambda \psi
$$

has emerged, with $J=\partial_{x}$ and $\mathcal{L}$ a self-adjoint operator. Since $J$ is not a one-to-one operator, classical linear stability results as those in [24] can not be applied. To overcome this difficult, recently Deconinck and Kapitula in [22] (see also Haragus and Kapitula [26]), have considered the similar problem

$$
\left.J \mathcal{L}\right|_{H_{0}} \psi=\lambda \psi
$$


in the closed subspace $H_{0}$ of mean zero,

$$
H_{0}=\left\{f \in L^{2}([0, L]) ; \int_{0}^{L} f(x) d x=0\right\} .
$$

Thus, a specific Krein-Hamiltonian index formula was deduced for concluding the linear stability of periodic profiles with the mean zero property. In particular, it was deduced the linear stability of periodic traveling waves of cnoidal type associated with equation (1.19) for $p=2$ (we also refer the reader to Bronski, Johnson and Kapitula in [17] and Deconinck and Nivala in [22]). We note, nevertheless, that for obtaining this specific result was necessary to know the periodic wave profile, as well as the quantity and multiplicity of the first eigenvalues associated to the Lamé problem

$$
-\Phi^{\prime \prime}+6 k^{2} \operatorname{sn}^{2}(x ; k) \Phi=\theta \Phi .
$$

Unfortunately, in our problem (1.17), this specific type of information can not be established.

We note that the spectral/orbital stability properties of periodic traveling waves in Hamiltonian equations that are first-order in time (e.g., the Korteweg-de Vries or the Schrödinger equations) have been very well-studied in recent years by using approaches different to those discussed above. See, for instance, Bronski and Johnson [16], Bronski, Johnson and Kapitula [17], [18], Bronski, Johnson and Zumbrun [19], Deconinck and Kapitula [21], Deconinck and Nivala [23], Haragus and Kapitula [26], Hur and Johnson [27], Jonhson [29], [30], and Kapitula and Promislow [31].

In section 5 below, we use the approaches in Angulo and Natali [11], Deconinck and Kapitula [22] and Haragus and Kapitula [26] for establishing the relevant result that the periodic profile $\varphi_{c}$ in (1.12) for the ILW equation is linearly stable for positive values of $c$ (see Remarks 4.4 and 5.9 below).

Our linear stability result determined in section 5 can be used to conclude the orbital stability of the periodic waves $\varphi_{c}$. To do so, we shall use an adaptation of the orbital stability analysis established by Andrade and Pastor in [7]. In our case, we need to consider periodic waves depending on the elliptic modulus $k$ instead of the wave speed $c$ in order to apply the mentioned approach. In this case, the stability analysis will be performance in the energy space (Hilbert space)

$$
\mathcal{W}=\left\{g \in L_{\text {per }}^{2}([0, L]) ;\|g\|_{\mathcal{W}}:=\left(\sum_{m=-\infty}^{+\infty}\left[1+\theta_{\delta}(m)\right]|\widehat{g}(m)|^{2}\right)^{1 / 2}<\infty\right\}
$$

where $\theta_{\delta}$ indicates the symbol associated to $\mathcal{M}_{\delta}$. In section 6 , we briefly describe the main arguments for obtaining our orbital result of the profile $\varphi_{c}$ by the periodic flow of the ILW-equation.

Our paper is organized as follows. In section 2 we present some notation and the definition of the Jacobi elliptic functions. Section 3 is devoted to the existence of periodic waves having the mean zero property. In section 4 , we present the required spectral property associated with the linear operator (1.18) by following the arguments in [10]. In section 5 , the linear stability of the periodic profile $\varphi_{c}$ in (1.12) will be shown. Finally, section 6 contains our orbital stability result. 


\section{Notation}

For $k \in(0,1)$, we define the normal elliptic integral of the first kind,

$$
u(x ; k)=\int_{0}^{x} \frac{d t}{\sqrt{\left(1-t^{2}\right)\left(1-k^{2} t^{2}\right)}}=\int_{0}^{\varphi} \frac{d \theta}{\sqrt{1-k^{2} \sin ^{2} \theta}}=F(\varphi ; k),
$$

with $x=\sin \varphi$. The number $k$ and $\varphi$ are called the modulus and the argument, respectively. For $x=1(\varphi=\pi / 2)$, the integral above is said to be complete. In this case, ones writes

$$
K(k)=\int_{0}^{1} \frac{d t}{\sqrt{\left(1-t^{2}\right)\left(1-k^{2} t^{2}\right)}}=\int_{0}^{\pi / 2} \frac{d \theta}{\sqrt{1-k^{2} \sin ^{2} \theta}} .
$$

Hence, $K(0)=\pi / 2$ and $K(1)=+\infty$. For $k$ fixed, $u=u(x ; k)$ is a strictly increasing function of variable $x$ (real). We define its inverse function by $x \equiv \operatorname{sn}(u ; k)$ (snoidal function). Then, we obtain the basic Jacobian elliptic functions cnoidal and dnoidal, defined by $\operatorname{cn}(u ; k) \equiv \sqrt{1-\operatorname{sn}^{2}(u ; k)}$ and $\operatorname{dn}(u ; k) \equiv \sqrt{1-k^{2} \operatorname{sn}^{2}(u ; k)}$ (see Byrd and Friedman [20] and Abramowitz and Segun [3]). Snoidal, cnoidal, and dnoidal have fundamental period $4 K(k), 4 K(k)$ and $2 K(k)$, respectively. Moreover, $\operatorname{sn}^{2}(u ; k)+\mathrm{cn}^{2}(u ; k)=1, k^{2} \operatorname{sn}^{2}(u ; k)+\operatorname{dn}^{2}(u ; k)=1, \operatorname{sn}(u ; 0)=\sin (u)$, $\operatorname{cn}(u ; 0)=\cos (u), \operatorname{sn}(u ; 1)=\tanh (u)$ and $\operatorname{cn}(u ; 1)=\operatorname{dn}(u, 1)=\operatorname{sech}(u)$. The Jacobi zeta function, $Z(u)=Z(u, k)$, it is defined for $u \in \mathbb{R}$ by

$$
Z(u)=\int_{0}^{u}\left[\operatorname{dn}^{2}(x ; k)-\frac{E(k)}{K(k)}\right] d x .
$$

This function is odd, with fundamental period $2 K(k)$. Moreover, $Z(\pi / 2, k)=0$ and $Z(m K)=0, m=0,1,2, \ldots$ For $u$ being a complex argument we refer the reader to formula 143.01 in [20]. In particular for $u=i x, x \in \mathbb{R}$, we obtain

$$
Z(i x, k)=i \frac{\operatorname{sn}\left(x ; k^{\prime}\right)}{\operatorname{cn}\left(x ; k^{\prime}\right)} \operatorname{dn}\left(x ; k^{\prime}\right)-i Z\left(x, k^{\prime}\right)-i \frac{\pi x}{2 K(k) K\left(k^{\prime}\right)},
$$

with $k^{\prime}=\sqrt{1-k^{2}}$.

\section{Existence of periodic waves}

This section is devoted to establish the property $(P 0)$ presented in the introduction. More precisely, we will construct a smooth curve of periodic waves with the mean zero property, $c \in I \mapsto \varphi_{c} \in H_{\text {per }}^{s}([0, L]) \cap H_{0}$, where the period $L$ and the wave speed $c$ satisfy some specific restrictions. Our arguments will follow Hirota's method, put forward in the works [36] and [37]. For the reader's convenience, we shall give a brief review of the method.

Indeed, let us assume the existence of $f: \mathbb{C} \times \mathbb{R} \rightarrow \mathbb{C}$ such that the profile

$$
u(x, t)=i \frac{\partial}{\partial x}\left[\ln \left(\frac{f(x+i \delta, t)}{f(x-i \delta, t)}\right)\right], \quad(x, t) \in \mathbb{R} \times \mathbb{R},
$$


satisfies equation (1.10), with $f(\cdot, t)$ analytic in a specific rectangle $R$ of the complex plane. To simplify the notation, we define $f_{+}(x, t)=f(x+i \delta, t)$ and $f_{-}(x, t)=$ $f(x-i \delta, t)$. From arguments in [37], there exists a constant $B$ such that the following bilinear equation is satisfied:

$$
\left[i D_{t}+\frac{i}{\delta} D_{x}-D_{x}^{2}+B\right] f_{+} \cdot f_{-}=0
$$

with

$$
D_{t}^{m} D_{x}^{n} a(x, t) \cdot b(x, t):=\left.\left(\partial_{t}-\partial_{t^{\prime}}\right)^{m}\left(\partial_{x}-\partial_{x^{\prime}}\right)^{n} a(x, t) b\left(x^{\prime}, t^{\prime}\right)\right|_{x=x^{\prime}, t=t^{\prime}} .
$$

In addition, we can deduce from (3.1) that

$$
F\left(D_{t}, D_{x}\right) f \cdot f=0
$$

where

$$
F\left(D_{t}, D_{x}\right) \equiv i\left(D_{t}+\frac{1}{\delta} D_{x}\right) \sinh \left(i \delta D_{x}\right)+\left(D_{x}^{2}-B\right) \cosh \left(i \delta D_{x}\right)
$$

Next, we consider $z=p x+w t$, where $p, w \in \mathbb{R}$ will be determined later. Suppose that $f$ has the following Jacobi theta profile (see [3]):

$$
f(x, t) \equiv \theta_{3}(z, q):=1+2\left[\sum_{n=1}^{+\infty} q^{n^{2}} \cos (2 n z)\right]=\sum_{n=-\infty}^{+\infty} q^{n^{2}} e^{2 i n z}
$$

where $q=e^{i \pi \tau}, \tau=i K\left(k^{\prime}\right) / K(k)$, and $K^{\prime}(k) \equiv K\left(\sqrt{1-k^{2}}\right)$ is the complete elliptic integral of the first kind. In general, $q=q(\tau)$ is the function called "nome" with $\operatorname{Im}(\tau)>0$. Substituting $f$ in $(3.2)$, one has

$$
\widetilde{F}_{0} \theta_{3}\left(2 z, q^{2}\right)+\widetilde{F}_{1} q^{-1 / 2} \theta_{2}\left(2 z, q^{2}\right)=0 .
$$

Here, $\theta_{2}$ represents the Jacobi theta function of the second kind. Moreover, one has

$$
\widetilde{F}_{m}=\sum_{n=-\infty}^{+\infty} F[2 i(2 n-m) w, 2 i(2 n-m) p] q^{n^{2}+(n-m)^{2}}, \quad m=0,1 .
$$

In order to prove that $f(x, t)=\theta_{3}(z, q)$ is a periodic solution related to the equation (1.10), it is sufficient to prove that $\tilde{F}_{0}=\tilde{F}_{1}=0$. To do so, we need to show that

$$
\frac{1}{\delta}\left(w+\frac{p}{\delta}\right) A_{0}^{\prime}-\frac{p^{2}}{\delta^{2}} A_{0}^{\prime \prime}-A_{0} B=0
$$

and

$$
\frac{1}{\delta}\left(w+\frac{p}{\delta}\right) A_{1}^{\prime}-\frac{p^{2}}{\delta^{2}} A_{1}^{\prime \prime}-A_{1} B=0
$$


where

$$
A_{0}=A_{0}(p ; q, \delta)=\sum_{n=-\infty}^{+\infty} q^{2 n^{2}} \cosh (4 n p \delta)=\theta_{3}\left(2 i p \delta, q^{2}\right)
$$

and

$$
A_{1}=A_{1}(p ; q, \delta)=\sum_{n=-\infty}^{+\infty} q^{n^{2}+(n-1)^{2}} \cosh [2(2 n-1) p \delta]=q^{1 / 2} \theta_{2}\left(2 i p \delta, q^{2}\right) .
$$

Here, $A_{0}^{\prime}$ and $A_{1}^{\prime}$ represent, respectively, the derivative of the parameters $A_{0}$ and $A_{1}$ with respect to $p$. Next, we consider fixed parameters $p, q$ and $\delta$ as above. Solving the system formed by (3.3) and (3.4), we get

$$
B=B(p ; q, \delta)=\frac{p^{2}}{\delta^{2}} \frac{A_{0}^{\prime} A_{1}^{\prime \prime}-A_{0}^{\prime \prime} A_{1}^{\prime}}{A_{0} A_{1}^{\prime}-A_{0}^{\prime} A_{1}}
$$

and

$$
w=w(p ; q, \delta)=-\frac{p}{\delta}+\frac{p^{2}}{\delta} \frac{A_{0} A_{1}^{\prime \prime}-A_{0}^{\prime \prime} A_{1}}{A_{0} A_{1}^{\prime}-A_{0}^{\prime} A_{1}}=-\frac{p}{\delta}+\frac{p^{2}}{\delta} \frac{\partial}{\partial p}\left\{\ln \left[W\left(A_{0}, A_{1}\right)\right]\right\},
$$

where $W\left(A_{0}, A_{1}\right)=A_{0} A_{1}^{\prime}-A_{0}^{\prime} A_{1}$ indicates the Wronskian of $A_{0}$ and $A_{1}$. Now, if we use some standard identities concerning the Jacobi elliptic functions (see [3] and $[20])$, we deduce that $f(x, t)=\theta_{3}(z, q)$ must satisfy the identity (3.2) provided that

$$
B=B(p ; q, \delta)=-p^{2}\left[\frac{\theta_{1}^{\prime \prime}(2 i p \delta, q)}{\theta_{1}(2 i p \delta, q)}-\frac{\theta_{1}^{\prime \prime \prime}(0, q)}{\theta_{1}^{\prime}(0, q)}\right]
$$

and

$$
w=w(p ; q, \delta)=-\frac{p}{\delta}+2 i p^{2} \frac{\theta_{1}^{\prime}(2 i p \delta, q)}{\theta_{1}(2 i p \delta, q)},
$$

where $\theta_{1}$ represents the Jacobi theta function of the first kind.

Similar arguments as above show that if we consider the change of variables $z \mapsto z / 2$, then

$$
B=B(p ; k, \delta)=-\frac{p^{2}}{4}\left[\frac{\theta_{1}^{\prime \prime}(i p \delta, q(k))}{\theta_{1}(i p \delta, q(k))}-\frac{\theta_{1}^{\prime \prime \prime}(0, q(k))}{\theta_{1}^{\prime}(0, q(k))}\right]
$$

and

$$
w=w(p ; k, \delta)=-\frac{p}{\delta}+i p^{2} \frac{\theta_{1}^{\prime}(i p \delta, q(k))}{\theta_{1}(i p \delta, q(k))} .
$$

Hence, we obtain that our hypothetical solution $u$ becomes

$$
\begin{aligned}
u(x, t) & =i \frac{\partial}{\partial x}\left\{\ln \left[\frac{\theta_{3}\left(\frac{1}{2}(z-i p \delta), q(k)\right)}{\theta_{3}\left(\frac{1}{2}(z+i p \delta), q(k)\right)}\right]\right\} \\
& =\frac{i p}{2}\left[\frac{\theta_{3}^{\prime}\left(\frac{1}{2}(z-i p \delta), q(k)\right)}{\theta_{3}\left(\frac{1}{2}(z-i p \delta), q(k)\right)}-\frac{\theta_{3}^{\prime}\left(\frac{1}{2}(z+i p \delta), q(k)\right)}{\theta_{3}\left(\frac{1}{2}(z+i p \delta), q(k)\right)}\right] .
\end{aligned}
$$

Moreover, $u$ represents a $L$-periodic function at the spatial variable with the natural choice of $p=2 \pi / L$. 
Next, we need to determine specific restrictions on the parameters $p, k$ and the minimal period $L$ in order to get a periodic function $u$. Indeed, let $k \in(0,1)$ be fixed. It is well known that the theta function $\theta_{3}(z, q(k))$ has simple zeros at the points

$$
z=\left(m+\frac{1}{2}\right) \pi+\left(n+\frac{1}{2}\right) \pi \tau, \quad m, n \in \mathbb{Z} .
$$

So, the right-hand side of (3.7) has infinitely many isolated singularities which we need to avoid. To overcome this difficulty, it is necessary to impose a convenient condition over the parameters $p, \delta$ and $k$, namely,

$$
0<p \delta<-i \pi \tau=\pi \frac{K\left(k^{\prime}\right)}{K(k)} .
$$

To do so, it suffices to consider $k \in(0,1)$ satisfying

$$
v(L, \delta, k):=\frac{2 \delta}{L} \frac{K(k)}{K\left(k^{\prime}\right)}<1 .
$$

Our next step is to present a convenient formula for the solution $u$ in (3.7). We consider the parameters $B$ and $w$ satisfying conditions in (3.5) and (3.6), respectively. Then, by using formula 16.43 .3 in [3] into (3.7), one has

$$
u(x, t)=\frac{2 K(k) i}{L}\left[Z\left(\frac{2 K(k)}{L}(\xi-i \delta) ; k\right)-Z\left(\frac{2 K(k)}{L}(\xi+i \delta) ; k\right)\right],
$$

where $\xi:=x-c t$ and $c:=-w / p$. Therefore, identity (3.10) determines a class of $L$-periodic functions which solves the ILW-equation (1.10) with wave speed $c$. Here, $Z$ represents the periodic Jacobi zeta function (see [3] and [20]).

Next, we will determine an expression for $c$. Indeed, in order to simplify the notation, let us define $d:=2 K(k) / L$. From the analysis above we obtain that

$$
c:=-\frac{w}{p}=\frac{1}{\delta}-i p \frac{\theta_{1}^{\prime}(i p \delta, q(k))}{\theta_{1}(i p \delta, q(k))}=\frac{1}{\delta}-\frac{2 \pi i}{L} \frac{\theta_{1}^{\prime}(2 \pi \delta i / L, q(k))}{\theta_{1}(2 \pi \delta i / L, q(k))} .
$$

Thus, by using formula 16.34 .1 in [3], we get

$$
c=\frac{1}{\delta}-2 d i\left[Z(2 d i \delta ; k)+\frac{\mathrm{cn}(2 d i \delta ; k) \mathrm{dn}(2 d i \delta ; k)}{\operatorname{sn}(2 d i \delta ; k)}\right],
$$

where sn, cn and dn denote the Jacobi elliptic functions snoidal, cnoidal and dnoidal, respectively. Hence, by considering $\xi=x-c t$ in $(2.10)$, we obtain the periodic traveling wave solution $\varphi_{c}$ in (1.12) related to the ILW equation. Moreover, by construction one has that $\varphi_{c} \in H_{0}$.

Next, by using formula 143.01 in [20], we can rewrite the profile $\varphi_{c}$ in terms of the Jacobi elliptic functions snoidal, cnoidal, and dnoidal as

$$
\begin{aligned}
\varphi_{c}(x)= & -2 d Z\left(d \delta ; k^{\prime}\right)-\frac{4 \delta \pi}{L^{2}} \frac{K(k)}{K\left(k^{\prime}\right)} \\
& +2 d \frac{\operatorname{dn}^{2}(d x ; k) \operatorname{cn}\left(d \delta ; k^{\prime}\right) \operatorname{sn}\left(d \delta ; k^{\prime}\right) \operatorname{dn}\left(d \delta ; k^{\prime}\right)}{1-\operatorname{dn}^{2}(d x ; k) \operatorname{sn}^{2}\left(d \delta ; k^{\prime}\right)} .
\end{aligned}
$$




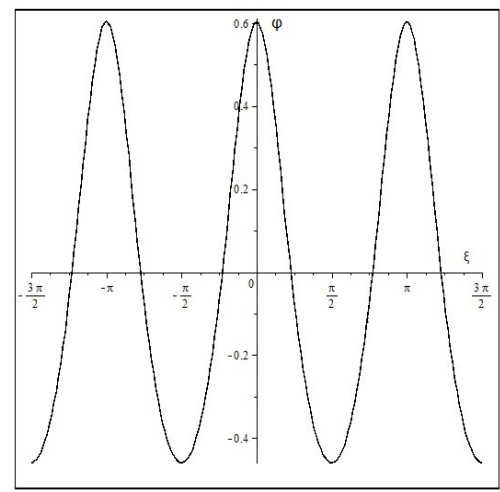

FiguRE 1. Function $\varphi_{c}$ in (3.12) with $L=\pi, \delta=1$ and $k=0.5$.

Moreover, formulas 143.02, 161.01 and 120.02 in [20] applied to the identity (3.11) give us a convenient expression for $c=c(k)$ :

$$
c=\frac{1}{\delta}-\frac{8 \pi \delta K(k)}{L^{2} K\left(k^{\prime}\right)}-2 d Z\left(2 \delta d ; k^{\prime}\right)-2 d \frac{\operatorname{cn}\left(2 \delta d ; k^{\prime}\right) \operatorname{dn}\left(2 \delta d ; k^{\prime}\right)}{\operatorname{sn}\left(2 \delta d ; k^{\prime}\right)} .
$$

Finally, from (3.9) we see that for $L$ and $\delta$ fixed, there exist an interval $\left(0, k_{1}\right) \subset$ $(0,1)$ such that $v(L, \delta, k)<1$, for all $k \in\left(0, k_{1}\right)$. Therefore, we have the following result, which guarantees the existence of periodic traveling wave solutions with the mean zero property related to the ILW equation.

Theorem 3.1. Let $L$ and $\delta$ be fixed. There exists $k_{1} \in(0,1)$ such that if $c=c(k)$ is defined as in (3.13), the map

$$
k \in\left(0, k_{1}\right) \rightarrow \varphi_{c(k)} \in H_{\text {per }}^{n}([0, L]) \cap H_{0}, \quad n \in \mathbb{N},
$$

is smooth with respect to $k \in\left(0, k_{1}\right)$ and it satisfies (1.11) with $A_{c}=A(k)=$ $\frac{1}{L} \int_{0}^{L} \varphi_{c(k)}^{2}(x) d x$.

In our linear and orbital stability analysis, we need to determine the sign of the derivative of $c(k)$ with respect to $k \in\left(0, k_{1}\right)$. For arbitrary values of $L$ and $\delta$, we are not able to find an analytical argument showing the behaviour of $c^{\prime}(k)$. However, numerical computations clearly show that $c=c(k)$ is a strictly increasing function over the interval $\left(0, k_{1}\right)$. For instance, by considering $L=\pi$ and $\delta=1$, we obtain the graphs of Figure 2, which show the behaviour of $c(k)$ and its derivative $c^{\prime}(k)$, respectively.

Moreover, by using formula in (3.13) and some numerical simulations, we obtain that $k_{1}$ in Theorem 3.1 is given by $k_{1} \approx 0,944085037$. Thus, for all $k \in\left(0, k_{1}\right)$, one has the basic condition $v(\pi, 1, k)<1$, such as is required in (3.9),

$$
c(0)=\lim _{k \rightarrow 0^{+}} c(k) \approx-1.07462944, \quad \text { and } \quad \lim _{k \rightarrow k_{1}^{-}} c(k)=+\infty .
$$



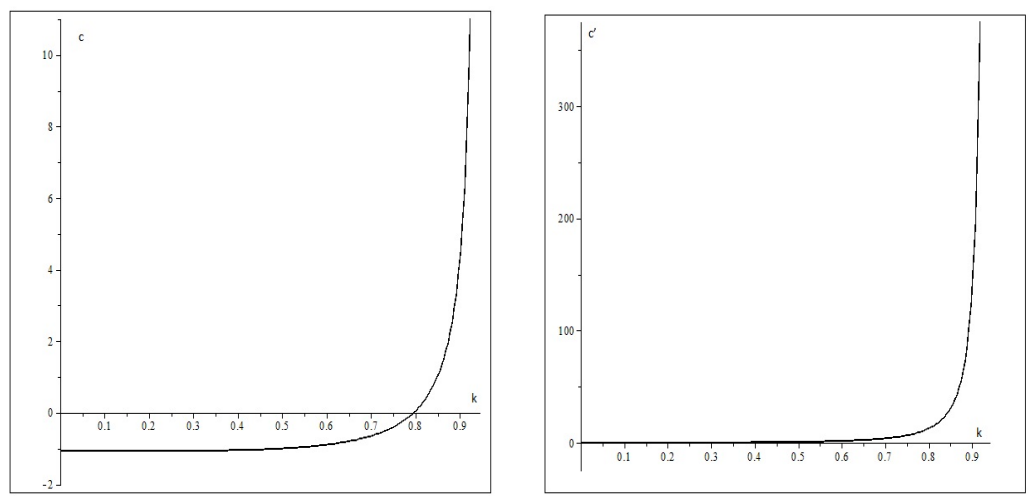

Figure 2. Behaviour of $c(k)$ and its derivative $c^{\prime}(k)$.

In addition, there exists a unique $k_{0} \approx 0.795178532$ such that

$$
c\left(k_{0}\right)=0 \quad \text { and } \quad c(k)>0, \quad \text { for all } k \in\left(k_{0}, k_{1}\right),
$$

that is, the wave speed $c$ is negative over the interval $\left(0, k_{0}\right)$.

\section{Spectral analysis}

In this section, we present the characterization of the nonpositive spectrum concerning the linearized operator $\mathcal{L}:=\mathcal{L}_{c, \delta}$ defined in (1.18). The main idea for this study will be to determine two specific spectral properties for $\mathcal{L}$, namely, that the kernel is one-dimensional with $\operatorname{ker}(\mathcal{L})=\left[\frac{d}{d x} \varphi_{c}\right]$ and the existence of a unique negative eigenvalue which is simple. Since the operator $\mathcal{L}$ is of non-local type, this analysis is not immediate. In this point, we will apply the theory of Angulo and Natali put forward in [11] for studying the stability of periodic traveling wave solutions associated to the general nonlinear dispersive model (1.5). The initial obstacle for applying Angulo and Natali's approach is that the periodic traveling wave profile $\psi=\psi_{\varsigma}$ related to equation (1.5) needs to be positive and to satisfy the equation

$$
\mathcal{M} \psi+\varsigma \psi-\psi^{2}=0
$$

Moreover, the wave speed $\varsigma$ must satisfy $\varsigma>-\inf _{r \in \mathbb{R}} \theta(r)$ in order to determine that $\mathcal{M}+\varsigma$ is a positive operator. In our analysis of existence established in last section, the traveling wave profile $\varphi_{c}$ in (1.12) has the mean zero property and the constant $A_{c}$ in (1.11) is not zero. In order to overcome this difficulty, we shall use that the ILW-equation has the Galilean invariance given by the transformation

$$
v(x, t)=u(x+2 \gamma t, t)-\gamma,
$$

where $\gamma \in \mathbb{R}$ is an arbitrary constant. The second obstacle in our analysis is to determine the required spectral properties associated to the linearized operator $\mathcal{L}$ 
in (1.18) for arbitrary values of $L$ and $\delta$. So, we shall restrict the analysis on a couple of specific values $L$ and $\delta$, namely, $L=\pi$ and $\delta=1$, respectively. However, numerical simulations enable us to conclude the same spectral properties for arbitrary values of $L$ and $\delta$.

In what follows, we establish some preliminaries definitions and results determined by Angulo and Natali in [10]. Moreover, we write $\varphi_{c}$ instead of $\varphi_{c(k)}$ in order to simplify the notation.

Definition 4.1. We say that a sequence $\alpha=\left(\alpha_{n}\right)_{n \in \mathbb{Z}} \subseteq \mathbb{R}$ is in the class $P F(2)$ discrete if

i) $\alpha_{n}>0$, for all $n \in \mathbb{Z}$,

ii) $\alpha_{n_{1}-m_{1}} \alpha_{n_{2}-m_{2}}-\alpha_{n_{1}-m_{2}} \alpha_{n_{2}-m_{1}} \geq 0$, for $n_{1}<n_{2}$ and $m_{1}<m_{2}$,

iii) $\alpha_{n_{1}-m_{1}} \alpha_{n_{2}-m_{2}}-\alpha_{n_{1}-m_{2}} \alpha_{n_{2}-m_{1}}>0$, if $n_{1}<n_{2}, m_{1}<m_{2}, n_{2}>m_{1}$, and $n_{1}<m_{2}$.

The definition above is a particular case of the continuous ones which appear in [4] (see also Karlin [33]): we say that a function $g: \mathbb{R} \rightarrow \mathbb{R}$ is in $P F(2)$-continuous if

i) $g(x)>0$, for all $x \in \mathbb{R}$,

ii) $g\left(x_{1}-y_{1}\right) g\left(x_{2}-y_{2}\right)-g\left(x_{1}-y_{2}\right) g\left(x_{2}-y_{1}\right) \geq 0$, for $x_{1}<x_{2}$ and $y_{1}<y_{2}$,

iii) strict inequality holds in (ii) whenever the intervals $\left(x_{1}, x_{2}\right)$ and $\left(y_{1}, y_{2}\right)$ intersect.

A sufficient condition for $g$ to belong to $P F(2)$-continuous appears if $g$ is smooth and logarithmically concave, namely,

$$
\frac{d^{2}}{d x^{2}} \log [g(x)]<0, \quad x \neq 0 .
$$

As examples of $P F(2)$-continuous functions, we have $Q_{0}(x)=\operatorname{sech}^{p}(x)$, for all $p>0$, and

$$
Q(x)=\frac{\sinh (\nu x)}{\sinh (\mu x)}, \quad 0<\nu<\mu .
$$

Hence, the sequences $\left(Q_{0}(n)\right)_{n \in \mathbb{Z}}$ and $(Q(n))_{n \in \mathbb{Z}}$ belong to the class $P F(2)$ discrete.

The main theorem in [11] is the following:

Theorem 4.2. Suppose that $\psi_{\varsigma}$ is an even positive solution of (1.11) with $A \equiv 0$, namely,

$$
\mathcal{M} \psi_{\varsigma}+\varsigma \psi_{\varsigma}-\psi_{\varsigma}^{2}=0
$$

such that $\left\{\widehat{\psi_{\varsigma}}(n)\right\}_{n \in \mathbb{Z}} \in P F(2)$ discrete. Then the self-adjoint operator $\mathcal{L}_{\varsigma}:=$ $\mathcal{M}+\varsigma-2 \psi_{\varsigma}$ possesses only one negative eigenvalue which is simple and zero is a simple eigenvalue with eigenfunction $\frac{d}{d x} \psi_{\varsigma}$. Moreover, its spectrum is bounded away from zero.

Our focus in the next lines is to apply Theorem 4.2 to determine the behaviour of the non positive spectrum associated to the linear operator $\mathcal{L}$ in (1.18). 
Theorem 4.3. Let $L=\pi$ and $\delta=1$ in (3.12)-(3.13). Consider $c=c(k)$ and $\varphi_{c}$, $k \in\left(0, k_{1}\right)$, as in Theorem 3.1. Then, $\mathcal{L}$ defined in $(1.18)$ is a self-adjoint operator such that $\operatorname{ker}(\mathcal{L})=\left[\frac{d}{d x} \varphi_{c}\right]$. In addition, $\mathcal{L}$ possess a unique negative eigenvalue which simple and the remainder of the spectrum is constituted by isolated real numbers which are bounded away from zero.

Proof. Initially, from the specific form of $\mathcal{L}$, we obtain from classical perturbation and spectral theories that $\mathcal{L}$ is a self-adjoint operator with a discrete spectrum (see $[10])$.

Now, in order to simplify the notation, we denote

$$
N(k):=\int_{0}^{L} \varphi_{c}^{2}(x) d x, \quad R(k):=\frac{N(k)}{L},
$$

and

$$
\begin{aligned}
& m_{1}:=2 d \operatorname{cn}\left(\delta d ; k^{\prime}\right) \operatorname{sn}\left(\delta d ; k^{\prime}\right) \operatorname{dn}\left(\delta d ; k^{\prime}\right), \\
& m_{2}:=\operatorname{sn}^{2}\left(\delta d ; k^{\prime}\right), \\
& m_{3}:=-2 d Z\left(\delta d ; k^{\prime}\right)-\frac{4 \delta \pi}{L^{2}} \frac{K(k)}{K\left(k^{\prime}\right)} .
\end{aligned}
$$

In our analysis, we shall consider $L$ and $\delta$ arbitrary but fixed. Thus, from (3.12), (4.1), (4.2) and (4.3), we get

$$
\varphi_{c}(x)=m_{1} \frac{\mathrm{dn}^{2}(d x ; k)}{1-m_{2} \operatorname{dn}^{2}(d x ; k)}+m_{3}
$$

and, consequently,

$$
N(k)
$$$$
(4.5)=m_{1}^{2} \int_{0}^{L} \frac{\operatorname{dn}^{4}(d x ; k)}{\left[1-m_{2} \operatorname{dn}^{2}(d x ; k)\right]^{2}} d x+2 m_{1} m_{3} \int_{0}^{L} \frac{\operatorname{dn}^{2}(d x ; k)}{1-m_{2} \operatorname{dn}^{2}(d x ; k)} d x+L m_{3}^{2} \text {. }
$$

Next, by using formula 410.04 in [20], we deduce

$$
\begin{aligned}
\int_{0}^{L} \frac{\mathrm{dn}^{2}(d x ; k)}{1-m_{2} \operatorname{dn}^{2}(d x ; k)} d x & =\frac{1}{d} \int_{0}^{d L} \frac{\mathrm{dn}^{2}(\zeta ; k)}{1-m_{2} \operatorname{dn}^{2}(\zeta ; k)} d \zeta \\
& =\frac{1}{d} \int_{0}^{2 K(k)} \frac{\mathrm{dn}^{2}(\zeta ; k)}{1-m_{2}+m_{2} k^{2} \operatorname{sn}^{2}(\zeta ; k)} d \zeta \\
& =\frac{2}{d\left(1-m_{2}\right)} \int_{0}^{K(k)} \frac{\mathrm{dn}^{2}(\zeta ; k)}{1-\alpha^{2} \operatorname{sn}^{2}(\zeta ; k)} d \zeta \\
& =\frac{2}{d\left(1-m_{2}\right)}\left[\frac{\pi\left(k^{2}-\alpha^{2}\right) \Lambda_{0}(\psi, k)}{2 \sqrt{\alpha^{2}\left(1-\alpha^{2}\right)\left(\alpha^{2}-k^{2}\right)}}\right]
\end{aligned}
$$


where

$$
\alpha^{2}=-\frac{m_{2} k^{2}}{1-m_{2}}<0, \quad m_{2} \neq 1, \quad \psi=\sin ^{-1}\left(\sqrt{\frac{\alpha^{2}}{\alpha^{2}-k^{2}}}\right),
$$

and $\Lambda_{0}$ indicates the Heuman lambda function defined by

$$
\Lambda_{0}(\psi, k)=\frac{2}{\pi}\left[E(k) F\left(\psi, k^{\prime}\right)+K(k) E\left(\psi, k^{\prime}\right)-K(k) F\left(\psi, k^{\prime}\right)\right],
$$

where

$$
E(k)=\int_{0}^{1} \sqrt{\frac{1-k^{2} t^{2}}{1-t^{2}}} d t, \quad E\left(\psi, k^{\prime}\right)=\int_{0}^{\psi} \sqrt{1-\left(1-k^{2}\right) \sin ^{2}(\theta)} d \theta
$$

and

$$
F\left(\psi, k^{\prime}\right)=\int_{0}^{\psi} \frac{d \theta}{\sqrt{1-\left(1-k^{2}\right) \sin ^{2}(\theta)}} .
$$

Therefore, formula 410.08 in [20] enables us to conclude

$$
\begin{aligned}
\int_{0}^{L} \frac{\operatorname{dn}^{4}(d x ; k)}{\left[1-m_{2} \operatorname{dn}^{2}(d x ; k)\right]^{2}} d x=\frac{1}{d} \int_{0}^{d L} \frac{\left[\operatorname{dn}^{2}(\zeta ; k)\right]^{2}}{\left[1-m_{2} \mathrm{dn}^{2}(\zeta ; k)\right]^{2}} d \zeta \\
=\frac{1}{d} \int_{0}^{2 K(k)} \frac{\left[1-k^{2} \operatorname{sn}^{2}(\zeta ; k)\right]^{2}}{\left[1-m_{2}+m_{2} k^{2} \operatorname{sn}^{2}(\zeta ; k)\right]^{2}} d \zeta \\
=\frac{2}{d\left(1-m_{2}\right)^{2}} \int_{0}^{K(k)} \frac{\left[1-k^{2} \operatorname{sn}^{2}(\zeta ; k)\right]^{2}}{\left[1-\alpha^{2} \operatorname{sn}^{2}(\zeta ; k)\right]^{2}} d \zeta \\
=\frac{2}{d\left(1-m_{2}\right)^{2}} \frac{1}{\alpha^{4}}\left[k^{4} K(k)+2 k^{2}\left(\alpha^{2}-k^{2}\right) \Pi\left(\alpha^{2}, k\right)+\left(\alpha^{2}-k^{2}\right)^{2} V_{2}\right],
\end{aligned}
$$

where

$$
\Pi\left(\alpha^{2}, k\right)=\frac{k^{2} K(k)}{k^{2}-\alpha^{2}}-\frac{\pi \alpha^{2} \Lambda_{0}(\psi, k)}{2 \sqrt{\alpha^{2}\left(1-\alpha^{2}\right)\left(\alpha^{2}-k^{2}\right)}}
$$

and

$$
\begin{aligned}
V_{2}= & \frac{1}{2\left(\alpha^{2}-1\right)\left(k^{2}-\alpha^{2}\right)}\left\{\frac{\left[2 k^{4} \alpha^{2}-2 k^{4}+\alpha^{4}\left(1-k^{2}\right)\right] K(k)}{k^{2}-\alpha^{2}}\right. \\
& \left.+\alpha^{2} E(k)-\frac{\pi\left(2 \alpha^{2} k^{2}+2 \alpha^{2}-\alpha^{4}-3 k^{2}\right) \alpha^{2} \Lambda_{0}(\psi, k)}{2 \sqrt{\alpha^{2}\left(1-\alpha^{2}\right)\left(\alpha^{2}-k^{2}\right)}}\right\} .
\end{aligned}
$$

Statements (4.1)-(4.12) give us

$$
\begin{aligned}
N(k)= & \frac{2 m_{1}^{2}}{d\left(1-m_{2}\right)^{2}} \frac{1}{\alpha^{4}}\left[k^{4} K(k)+2 k^{2}\left(\alpha^{2}-k^{2}\right) \Pi\left(\alpha^{2}, k\right)+\left(\alpha^{2}-k^{2}\right)^{2} V_{2}\right] \\
& +\frac{2 m_{1} m_{3}}{d\left(1-m_{2}\right)}\left[\frac{\pi\left(k^{2}-\alpha^{2}\right) \Lambda_{0}(\psi, k)}{\sqrt{\alpha^{2}\left(1-\alpha^{2}\right)\left(\alpha^{2}-k^{2}\right)}}\right]+L m_{3}^{2} .
\end{aligned}
$$


Next, by considering the specific values of $L=\pi, \delta=1$, we obtain for each $k \in\left(0, k_{1}\right)$, the existence of $a=a(k)>0$ such that

$$
a^{2}+c a-R=0 \text {. }
$$

Thus, one has

$$
a=\frac{-c+\sqrt{c^{2}+4 R}}{2} .
$$

Moreover, by using that

$$
\min _{x \in[0, L]} \varphi_{c}(x)=\varphi_{c}\left(\frac{L}{2}\right),
$$

we find, by using numerical simulations (see Figure 3 below), that

$$
a(k)>-\varphi_{c}\left(\frac{L}{2}\right), \quad \text { for all } k \in\left(0, k_{1}\right) .
$$

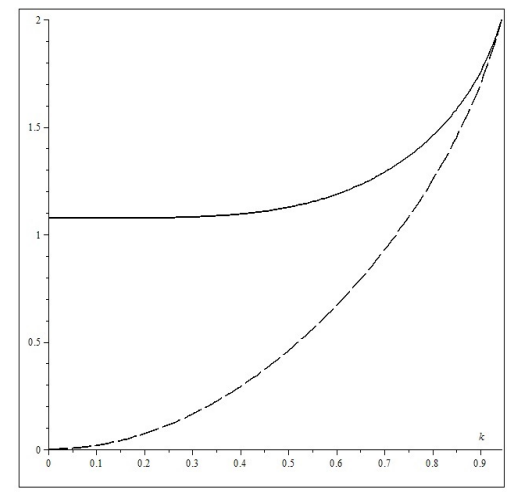

Figure 3. Consider $L=\pi$ and $\delta=1$ in (3.12)-(3.13). The continuous line gives us the behaviour of the function $a=a(k)$ in (4.15) for $k \in\left(0, k_{1}\right)$. The dashed line shows the behaviour of $-\varphi_{c}(L / 2)$ for $k \in\left(0, k_{1}\right)$.

Now, let us define the velocity parameter $\varsigma=\varsigma(k)$ by

$$
\varsigma:=c+2 a=\sqrt{c^{2}+4 R}>0
$$

and consider the translation function $\phi_{\varsigma}:=a+\varphi_{c}$. By using (4.16), we conclude that $\phi_{\varsigma}>0$. Moreover, since $\varphi_{c}$ is an even $L$-periodic function, one has that $\phi_{\varsigma}$ is also an even $L$-periodic function. Now, we claim that $\phi_{\varsigma}$ satisfies equation (1.11) with $A \equiv 0$. Indeed, since $\mathcal{M}_{\delta}\left(\varphi_{c}+\beta\right)=\mathcal{M}_{\delta} \varphi_{c}$, for all $\beta \in \mathbb{R}$, it follows from (1.11) and (4.14) that

$$
\begin{aligned}
-\mathcal{M}_{\delta} \phi_{\varsigma}-\varsigma \phi_{\varsigma}+\phi_{\varsigma}^{2} & =-\mathcal{M}_{\delta} \phi_{\varsigma}-c \phi_{\varsigma}-2 a \phi_{\varsigma}+\phi_{\varsigma}^{2} \\
& =-\mathcal{M}_{\delta} \varphi_{c}-c\left(\varphi_{c}+a\right)-2 a\left(\varphi_{c}+a\right)+\left(\varphi_{c}+a\right)^{2} \\
& =-\mathcal{M}_{\delta} \varphi_{c}-c \varphi_{c}+\varphi_{c}^{2}-\left(c a+a^{2}\right)=0 .
\end{aligned}
$$


In what follows, we will verify that for all $k \in\left(0, k_{1}\right),\left\{\widehat{\phi_{\varsigma}}(n)\right\}_{n \in \mathbb{Z}} \in P F(2)$ discrete. We recall that if $k \in\left(0, k_{1}\right)$ one has that the analytic condition in (3.9) is verified $(L=\pi$ and $\delta=1)$. Applying formula 905.01 in [20] into (1.12), we obtain

$$
\begin{aligned}
\varphi_{c}(x) & =\frac{2 \pi i}{L} \sum_{m=1}^{+\infty}\left[\frac{\sin \left(\frac{2 m \pi}{L}(x-i \delta)\right)}{\sinh \left(m \pi K\left(k^{\prime}\right) / K(k)\right)}-\frac{\sin \left(\frac{2 m \pi}{L}(x+i \delta)\right)}{\sinh \left(m \pi K\left(k^{\prime}\right) / K(k)\right)}\right] \\
& =\frac{4 \pi}{L} \sum_{m=1}^{+\infty} \frac{\sinh (2 m \pi \delta / L)}{\sinh \left(m \pi K\left(k^{\prime}\right) / K(k)\right)} \cos (2 m \pi x / L)
\end{aligned}
$$

that is,

$$
\phi_{\varsigma}(x)=a+\frac{4 \pi}{L} \sum_{m=1}^{+\infty} \frac{\sinh (2 m \pi \delta / L)}{\sinh \left(m \pi K\left(k^{\prime}\right) / K(k)\right)} \cos (2 m \pi x / L) .
$$

So, the periodic Fourier transform of $\phi_{\varsigma}$ is expressed by $\widehat{\phi_{\varsigma}}(0)=a$ and

$$
\widehat{\phi_{\varsigma}}(m)=\frac{2 \pi}{L} \frac{\sinh (2 m \pi \delta / L)}{\sinh \left(m \pi K\left(k^{\prime}\right) / K(k)\right)}, \quad \text { for all } m \in \mathbb{Z}-\{0\} .
$$

Now, we consider

$$
\nu:=\frac{2 \pi \delta}{L} \quad \text { and } \quad \mu:=\frac{\pi K\left(k^{\prime}\right)}{K(k)} .
$$

Then, from (3.9), we have $0<\nu<\mu$. On the other hand, by considering

$$
Q(x):=\frac{\sinh (\nu x)}{\sinh (\mu x)}, \quad x \neq 0,
$$

we see that

$$
\frac{d^{2}}{d x^{2}}[\log (Q(x))]<0, \quad \forall x \neq 0 .
$$

Therefore, it follows that $Q \in P F(2)$-continuous (see [4]). In addition, we obtain the following specific calculation which will be useful later:

$$
\lim _{x \rightarrow 0} \frac{2 \pi}{L} \frac{\sinh (2 \pi \delta x / L)}{\sinh \left(\pi K\left(k^{\prime}\right) x / K(k)\right)}=\frac{4 \pi \delta K(k)}{L^{2} K\left(k^{\prime}\right)} .
$$

Next, Figure 4 shows that the function

$$
a(k)-\frac{2 \pi}{L} v(L, \delta, k) \equiv a(k)-2 v(\pi, 1, k), \quad k \in\left(0, k_{1}\right)
$$

is strictly positive.

Therefore, we obtain for all $k \in\left(0, k_{1}\right)$, the following key inequality for $a=a(k)$ :

$$
a>\frac{4 \pi \delta K(k)}{L^{2} K\left(k^{\prime}\right)}=\frac{2 \pi}{L} v(L, \delta, k) .
$$




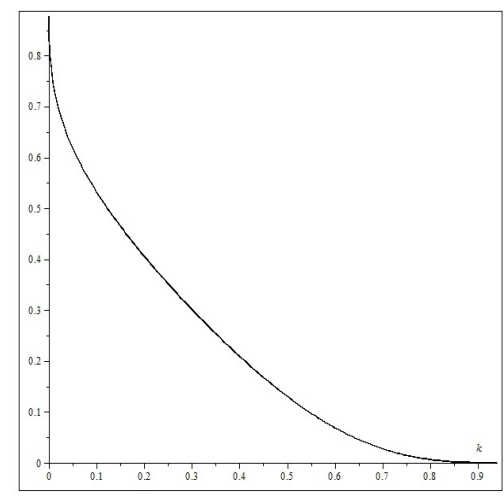

Figure 4.

Hence, statements (4.20)-(4.23) allow us to define a smooth function $\tau: \mathbb{R} \rightarrow \mathbb{R}$

$$
\tau(x):=\frac{2 \pi Q(x)}{L}, \quad \forall x \in(-\infty,-1] \cup[1,+\infty)
$$

such that $\tau(0)=a$ and $\tau$ in $(-1,1)$, and such that $\tau \in P F(2)$ continuous. Therefore, we can conclude that

$$
\left\{\widehat{\phi_{\varsigma}}(m)\right\}_{m \in \mathbb{Z}} \in P F(2) \text { discrete. }
$$

Hence, from Theorem 4.2 we obtain that the linear operator $\mathcal{L}_{\varsigma, \delta}=\mathcal{M}_{\delta}+\varsigma-2 \phi_{\varsigma}$ admits exactly one negative eigenvalue which is simple and zero is also a simple eigenvalue whose correspondent eigenfunction is $\frac{d}{d x} \phi_{\varsigma}$.

Finally, we analyze our linearized operator $\mathcal{L}$ in (1.18). Indeed, since

$$
\begin{aligned}
\mathcal{L}_{\varsigma, \delta} & =\mathcal{M}_{\delta}+\varsigma-2 \phi_{\varsigma}=\mathcal{M}_{\delta}+(c+2 a)-\left(2 \varphi_{c}+2 a\right) \\
& =\mathcal{M}_{\delta}+c-2 \varphi_{c}=\mathcal{L},
\end{aligned}
$$

we obtain

$$
\operatorname{ker}(\mathcal{L})=\operatorname{ker}\left(\mathcal{L}_{\varsigma, \delta}\right)=\left[\frac{d}{d x} \phi_{\varsigma}\right]=\left[\frac{d}{d x} \varphi_{c}\right], \quad \text { and } \quad n(\mathcal{L})=1
$$

This finishes the proof of the theorem.

Remark 4.4. We have some technical difficulties to determine that (4.16) holds for arbitrary values of $L$ and $\delta$ (in our approach, we need to use numerical simulations related to specific values of $L$ and $\delta$ ). However, Maple 16 software enables us to conclude that (4.16) is valid for general values of $L$ and $\delta$ provided that the analytic condition in (3.9) is verified. As a consequence, the results in Theorem 4.3 can be established for arbitrary values of $L$ and $\delta$. 


\section{Linear stability for the ILW equation}

In this section we establish our linear stability result for the mean zero traveling wave $\varphi_{c}$ in (1.12). For the convenience of the reader, we shall give some definitions and sufficient conditions for obtaining our linear stability results (see [22] and [26]).

We start with the following definitions associated to the restricted operator $\left.\partial_{x} \mathcal{L}\right|_{H_{0}}$, where $\mathcal{L}=\mathcal{L}_{c, \delta}$ is given in (1.18) and $H_{0}$ is defined in (1.21).

Definition 5.1. We define

1) $k_{r}$ as the number of real positive eigenvalues (counting multiplicities) of the operator $\left.\partial_{x} \mathcal{L}\right|_{H_{0}}$;

2) $k_{c}$ as the number of complex eigenvalues with positive real part (counting multiplicities) of the operator $\left.\partial_{x} \mathcal{L}\right|_{H_{0}}$;

3 ) the imaginary part of a linear operator $B$ with domain $D(B)$ as the linear operator $\operatorname{Im}(B) u \equiv \operatorname{Im}(B u), u \in D(B)$.

From the previous definition, we see that since $\operatorname{Im}(\mathcal{L}) \equiv 0$, then $k_{c}$ is an even integer. Next, if $\mathcal{B}$ is a self-adjoint operator, we denote by $n(\langle w, \mathcal{B} w\rangle)$ the dimension of the maximal subspace such that $\langle w, \mathcal{B} w\rangle<0$ (also called the Morse index of $\mathcal{B})$. Moreover, let $\lambda$ be an eigenvalue for $\partial_{x} \mathcal{L}$ and consider its corresponding eigenspace $E_{\lambda}$. The eigenvalue $\lambda$ is said to have negative Krein signature if

$$
k_{i}^{-}(\lambda):=n\left(\left\langle w,\left.\left(\left.\mathcal{L}\right|_{H_{0}}\right)\right|_{E_{\lambda}} w\right\rangle\right) \geq 1
$$

if $k_{i}^{-}(\lambda)=0$, the eigenvalue $\lambda$ is said to have positive Krein signature. If $\lambda$ is a geometrically and algebraically simple eigenvalue related to $\partial_{x} \mathcal{L}$ with eigenfunction $\psi_{\lambda}$, then $E_{\lambda}=\left[\psi_{\lambda}\right]$, and so

$$
k_{i}^{-}(\lambda)= \begin{cases}0, & \text { if }\left\langle\psi_{\lambda},\left(\left.\mathcal{L}\right|_{H_{0}}\right) \psi_{\lambda}\right\rangle>0, \\ 1, & \text { if }\left\langle\psi_{\lambda},\left(\left.\mathcal{L}\right|_{H_{0}}\right) \psi_{\lambda}\right\rangle<0\end{cases}
$$

The total Krein signature is given by $k_{i}^{-}:=\sum_{\lambda \in i \mathbb{R} \backslash\{0\}} k_{i}^{-}(\lambda)$. Since $\operatorname{Im}(\mathcal{L})=0$, we obtain that $k_{i}^{-}$is an even integer.

Definition 5.2. The Hamiltonian-Krein index associated to the operator $\partial_{x} \mathcal{L}$ is the following non-negative integer:

$$
\mathcal{K}_{\text {Ham }}=k_{r}+k_{c}+k_{i}^{-} .
$$

Next, let us consider the quantity

$$
\mathcal{I}=\left\langle\mathcal{L}^{-1} 1,1\right\rangle .
$$

We note that for any $f \in \operatorname{ker}(\mathcal{L})^{\perp}$, the quantity $\left\langle\mathcal{L}^{-1} f, f\right\rangle$ is always independent of $h \in \mathcal{L}^{-1} f$. Now, we denote by $\mathcal{D}$ the following determinant:

$$
\mathcal{D}=\frac{1}{\left\langle\mathcal{L}^{-1} 1,1\right\rangle}\left|\begin{array}{cc}
\left\langle\mathcal{L}^{-1} \varphi_{c}, \varphi_{c}\right\rangle & \left\langle\mathcal{L}^{-1} \varphi_{c}, 1\right\rangle \\
\left\langle\mathcal{L}^{-1} \varphi_{c}, 1\right\rangle & \left\langle\mathcal{L}^{-1} 1,1\right\rangle
\end{array}\right| .
$$

Thus, from [22] and [26] we have the following result. 
Theorem 5.3. Suppose that $\operatorname{ker}(\mathcal{L})=\left[\frac{d}{d x} \varphi_{c}\right]$. If $\mathcal{I}$ and $\mathcal{D}$ are both non-zero, the following identity holds:

$$
\mathcal{K}_{\text {Ham }}=n(\mathcal{L})-n(\mathcal{I})-n(\mathcal{D}) .
$$

Now, by considering $\mathcal{A}=\mathcal{I}$ or $\mathcal{A}=\mathcal{D}$, we have $n(\mathcal{A})=0 \Leftrightarrow \mathcal{A}>0$, and $n(\mathcal{A})=1 \Leftrightarrow \mathcal{A}<0$. An immediate consequence of Theorem 5.3 and Definition 1.1 is the following.

Corollary 5.4. Under the assumptions of Theorem 5.3, if $k_{c}=k_{r}=k_{i}^{-}=0$, then the periodic wave $\varphi_{c}$ is linearly stable. In addition, if $\mathcal{K}_{\mathrm{Ham}}=1$, then the periodic wave is linearly unstable.

Proof. The first part of the corollary is an immediate consequence of Theorem 2.7 in [22] (see also [26]). Now, if $\mathcal{K}_{\text {Ham }}=1$ we deduce that $k_{r}=1$ since $k_{c}$ and $k_{i}^{-}$ are even nonnegative integers. Then, the spectral problem in (1.20) has a positive eigenvalue. This last fact enables us to deduce the linear instability of the periodic wave $\varphi_{c}$.

Next, we establish our linear stability result associated to the periodic traveling wave $\varphi_{c}$ in (3.12). Since our study will be based on Theorem 5.3, the value of $\mathcal{K}_{\text {Ham }}$ must be calculated. From Theorem 4.3, we have that $n(\mathcal{L})=1$. Now, we will prove that $n(\mathcal{D})=1$ and $n(\mathcal{I})=0$, only for $c>0$ (by technical reasons associated to the sign of $\left.c^{\prime}(k)\right)$. Next, we obtain some convenient expressions for $\mathcal{I}$ and $\mathcal{D}$. More precisely, we have (see propositions below) the following formulas:

$$
\mathcal{I}=\left\langle\mathcal{L}^{-1} 1,1\right\rangle=\frac{L^{2}}{c L+2 \frac{\partial}{\partial c}\left[\int_{0}^{L} \varphi_{c}^{2}(x) d x\right]},
$$

and

$$
\mathcal{D}=-\frac{1}{2} \frac{1}{\mathcal{I}} \frac{\partial}{\partial c} \int_{0}^{L} \varphi_{c}^{2}(x) d x .
$$

In our analysis, we deduce that $\frac{\partial}{\partial c} \int_{0}^{L} \varphi_{c}^{2}(x) d x>0$, and consequently, we get $\mathcal{I}>0$ and $\mathcal{D}<0$. Thus, one has, respectively, $n(\mathcal{I})=0$ and $n(\mathcal{D})=1$. Therefore, from Theorem 4.3 and Theorem 5.3, we conclude that $\mathcal{K}_{\text {Ham }}=0$. Consequently, we deduce the linear stability of the wave $\varphi_{c}$ (see Corollary 5.4) according with next result.

Theorem 5.5. Consider $c>0$. The periodic traveling waves $\varphi_{c}$ in (3.12) is linearly stable for the ILW equation.

The focus of the following propositions will be to show that $\mathcal{I}>0$ and $\mathcal{D}<0$. We recall that, for convenience, we are considering $L=\pi$ and $\delta=1$. We start by establishing the following key result. 
Proposition 5.6. For all $c>0$ one has $\frac{\partial}{\partial c} \int_{0}^{L} \varphi_{c}^{2}(x) d x>0$.

Proof. Initially we have, for $N(k)=\int_{0}^{L} \varphi_{c(k)}^{2}(x) d x$, the relation

$$
\frac{\partial}{\partial c}\left[\int_{0}^{L} \varphi_{c}^{2}(x) d x\right]=\frac{d k}{d c} \frac{\partial}{\partial k}\left[\int_{0}^{L} \varphi_{c(k)}^{2}(x) d x\right] \equiv \frac{d k}{d c} N^{\prime}(k) .
$$

Thus, since $c^{\prime}(k)>0$, for all $k \in\left(k_{0}, k_{1}\right)$ (see (3.15)), we only need to establish the sign of $N^{\prime}(k)$. Before that, it is necessary to handle the quantity $N(k)$ in $(4.5)$ for obtaining a convenient expression in our calculations. Indeed, from (4.17) and from the Plancherel theorem we obtain

$$
N(k)=L \sum_{m=-\infty}^{+\infty}\left|\widehat{\varphi_{c}}(m)\right|^{2}=\frac{8 \pi^{2}}{L} \sum_{m=1}^{+\infty} \frac{[\sinh (2 m \pi \delta / L)]^{2}}{\left[\sinh \left(m \pi K\left(k^{\prime}\right) / K(k)\right)\right]^{2}},
$$

for all $k \in\left(0, k_{1}\right)$. So, one can take the first derivative with respect to $k \in\left(k_{0}, k_{1}\right)$ in (5.6) to deduce

$$
N^{\prime}(k)=-\frac{16 \pi^{3}}{L} \sum_{m=1}^{+\infty}\left\{\frac{m\left[\sinh \left(\frac{2 m \pi \delta}{L}\right)\right]^{2}\left[\cosh \left(\frac{m \pi K\left(k^{\prime}\right)}{K(k)}\right)\right]\left[\frac{d}{d k}\left[\frac{K\left(k^{\prime}\right)}{K(k)}\right]\right]}{\left[\sinh \left(\frac{m \pi K\left(k^{\prime}\right)}{K(k)}\right)\right]^{3}}\right\} .
$$

Since

$$
\frac{d}{d k}\left[\frac{K\left(k^{\prime}\right)}{K(k)}\right]=\frac{[E(k)-K(k)] K\left(k^{\prime}\right)+K(k) E\left(k^{\prime}\right)}{k\left(k^{2}-1\right) K(k)^{2}}<0,
$$

for all $k \in(0,1)$, we obtain immediately that

$$
N^{\prime}(k)>0, \quad \text { for all } k \in\left(k_{0}, k_{1}\right) .
$$

This finishes the proof.

Remark 5.7. From Proposition 5.6 and numerical calculations made in section 2 (see $(3.15))$, we see that $N^{\prime}(k)>0$ for every $k \in\left(0, k_{1}\right)-\left\{k_{0}\right\}$. So, we have

$$
\frac{d}{d c}\left\|\varphi_{c}\right\|^{2}>0, \quad \text { for every } c \neq 0 \text {. }
$$

Next we establish the formulas (5.3) and (5.4).

Proposition 5.8. For every $c>0$, we have that $\mathcal{I}>0$. In particular, $n(\mathcal{I})=0$.

Proof. Since $f \equiv 1 \in H_{\mathrm{per}}^{s}([0, L])$, for all $s \geq 0$, and $\mathcal{M}_{\delta}(1)=0$, we get

$$
\mathcal{L}(1)=\mathcal{M}_{\delta}(1)+c-2 \varphi_{c}=c-2 \varphi_{c}
$$

Thus, since $\operatorname{ker}(\mathcal{L})=\left[\frac{d}{d x} \varphi_{c}\right], \frac{d}{d x} \varphi_{c} \perp 1$ and $\frac{d}{d x} \varphi_{c} \perp \varphi_{c}$, one has from (5.9) that

$$
1=c \mathcal{L}^{-1} 1-2 \mathcal{L}^{-1} \varphi_{c}
$$


Then

$$
c\left\langle\mathcal{L}^{-1} 1,1\right\rangle=\langle 1,1\rangle+2\left\langle\mathcal{L}^{-1} \varphi_{c}, 1\right\rangle .
$$

Now, since $c>0$, we get

$$
\left\langle\mathcal{L}^{-1} 1,1\right\rangle=\frac{L}{c}+\frac{2\left\langle\mathcal{L}^{-1} \varphi_{c}, 1\right\rangle}{c} .
$$

Next, by differentiating identity (1.11) with respect to $c$, we obtain

$$
\mathcal{L}\left(\frac{\partial}{\partial c} \varphi_{c}\right)=-\varphi_{c}-\frac{1}{L} \frac{d}{d c}\left\|\varphi_{c}\right\|^{2}
$$

Applying the operator $\mathcal{L}^{-1}$ to both sides of (5.12), we deduce

$$
\frac{\partial}{\partial c} \varphi_{c}=-\mathcal{L}^{-1} \varphi_{c}-\frac{1}{L} \frac{d}{d c}\left\|\varphi_{c}\right\|^{2} \mathcal{L}^{-1} 1 .
$$

Hence, since $\varphi_{c}$ has the mean zero property, we have

$$
\left\langle\frac{\partial}{\partial c} \varphi_{c}, 1\right\rangle=\frac{\partial}{\partial c} \int_{0}^{L} \varphi_{c}(x) d x=0,
$$

and so, by combining (5.13) and (5.14), it follows that

$$
\left\langle\mathcal{L}^{-1} \varphi_{c}, 1\right\rangle+\frac{1}{L} \frac{d}{d c}\left\|\varphi_{c}\right\|^{2}\left\langle\mathcal{L}^{-1} 1,1\right\rangle=0 .
$$

Therefore, from (5.11) and (5.15) we obtain the equality

$$
\left\langle\mathcal{L}^{-1} 1,1\right\rangle+\frac{2}{L c} \frac{d}{d c}\left\|\varphi_{c}\right\|^{2}\left\langle\mathcal{L}^{-1} 1,1\right\rangle=\frac{L}{c} .
$$

Finally, since $\frac{d}{d c}\left\|\varphi_{c}\right\|^{2}>0$ (Proposition 5.6), we get

$$
\mathcal{I}=\left\langle\mathcal{L}^{-1} 1,1\right\rangle=\frac{L^{2}}{c L+2 \frac{d}{d c}\left\|\varphi_{c}\right\|^{2}} .
$$

Therefore, we obtain the formula in (5.3). Moreover, from the assumption about $c$ and Proposition 5.6, we have immediately that $\mathcal{I}>0$. This finishes the proof.

Remark 5.9. From (5.16), we note that our imposition about the positiveness of $c$ in Proposition 5.8 has only technical reasons. In fact, if $c<0$, the study of the behaviour of $\mathcal{I}$ will depend on exhaustive numerical calculations. Additional calculations made in Maple 16 enable us to say that $\mathcal{I}>0$, for all $c \neq 0(k \in$ $\left.\left(0, k_{1}\right)-\left\{k_{0}\right\}\right)$.

Proposition 5.10. For $c>0$, we obtain $\mathcal{D}<0$. In particular, $n(\mathcal{D})=1$. 
Proof. We start our proof by determining convenient expressions for each term of $\mathcal{D}$ in (5.2). Indeed, from (5.11) and (5.16), we deduce

$$
\left\langle\mathcal{L}^{-1} \varphi_{c}, 1\right\rangle=\frac{c\left\langle\mathcal{L}^{-1} 1,1\right\rangle}{2}-\frac{L}{2}=-\frac{L \frac{d}{d c}\left\|\varphi_{c}\right\|^{2}}{c L+2 \frac{d}{d c}\left\|\varphi_{c}\right\|^{2}} .
$$

Hence, by using identities $(5.10),(5.17)$ and the fact that $\varphi_{c} \in H_{0}$, we obtain

$$
\left\langle\mathcal{L}^{-1} \varphi_{c}, \varphi_{c}\right\rangle=\frac{c}{2}\left\langle\mathcal{L}^{-1} 1, \varphi_{c}\right\rangle=-\frac{c L \frac{d}{d c}\left\|\varphi_{c}\right\|^{2}}{2 c L+4 \frac{d}{d c}\left\|\varphi_{c}\right\|^{2}} .
$$

Therefore, since $\mathcal{I} \neq 0$ (Proposition 5.8) it follows from (5.16), (5.17) and (5.18) that

$$
\begin{aligned}
\mathcal{D} & =\frac{1}{\mathcal{I}}\left[\left\langle\mathcal{L}^{-1} \varphi_{c}, \varphi_{c}\right\rangle-\frac{\left\langle\mathcal{L}^{-1} \varphi_{c}, 1\right\rangle^{2}}{\left\langle\mathcal{L}^{-1} 1,1\right\rangle}\right] \\
& =-\frac{1}{\mathcal{I}}\left[\frac{c L \frac{d}{d c}\left\|\varphi_{c}\right\|^{2}}{2 c L+4 \frac{d}{d c}\left\|\varphi_{c}\right\|^{2}}-\frac{\left[\frac{d}{d c}\left\|\varphi_{c}\right\|^{2}\right]^{2}}{c L+2 \frac{d}{d c}\left\|\varphi_{c}\right\|^{2}}\right]=-\frac{1}{2} \frac{1}{\mathcal{I}} \frac{d}{d c}\left\|\varphi_{c}\right\|^{2} .
\end{aligned}
$$

Therefore, we obtain the formula in (5.4) and from Propositions 5.6 and 5.8, we have $\mathcal{D}<0$. The proof of the proposition is completed.

Remark 5.11. From (1.17) and the fact that $\mathcal{L}=\mathcal{L}_{\varsigma, \delta}$, we deduce that the positive and periodic wave $\phi_{\varsigma}$ is also linearly stable.

\section{Orbital stability for the ILW-equation}

In the last section, we have proved that the Krein-Hamiltonian index $\mathcal{K}_{\text {Ham }}$ associated to the linear operator $\partial_{x} \mathcal{L}$ is zero, and thus the linear stability of the periodic traveling wave $\varphi_{c}$ was obtained. The next step of the theory is to obtain information about the orbital stability of these periodic profiles. Indeed, by using arguments in [22], [24], [25], and Chapter 5.2.2 of [31], we will deduce that $\varphi_{c}$ is a local minimizer of a constrained energy functional, and so the orbital stability of these periodic waves can be deduced. The information obtained in Theorem 4.3 and Proposition 5.6 are the basis of our analysis.

Now, in some works in the current literature (for instance, [8], [12], [14], [25], [24], [28], [39]), we see that the orbital stability of periodic traveling waves related to the general model (1.11) were determined provided that the constant $A_{c}$ does not depend on the wave speed $c$ (we believe that the mentioned approaches can not be directly used to conclude the orbital stability of periodic profiles when $A_{c}$ is a function of $c$ ). Thus, our purpose will be to apply the recent development in Andrade and Pastor [7] to overcome this difficulty and then, we obtain the orbital stability of the profile $\varphi_{c}$ in (1.12) for every $c \neq 0$. 
We start our study by presenting the formal definition of orbital stability.

Definition 6.1. We say that the periodic wave $\varphi_{c}$ in (1.12) is orbitally stable with respect to (1.10) in the space $\mathcal{W}$ in (1.22), if for all $\varepsilon>0$, there exists $\delta>0$ such that if $u_{0} \in H_{\text {per }}^{s}([0, L]) \cap \mathcal{W}, s>3 / 2$, with $\left\|u_{0}-\varphi_{c}\right\|_{\mathcal{W}}<\delta$ and $u(t)$ is the solution of (1.10) with $u(0)=u_{0}$, then for all $t \in \mathbb{R}$, one has

$$
\inf _{r \in \mathbb{R}}\left\|u(t)-\varphi_{c}(\cdot+r)\right\|_{\mathcal{W}}<\varepsilon
$$

Otherwise, the periodic wave $\varphi_{c}$ is said to be orbitally unstable.

Definition 6.1 forces us to obtain some information about the global wellposedness related to the ILW-equation. This is the content of the following result.

Theorem 6.2. Consider $u_{0} \in H_{\mathrm{per}}^{s}([0, L])$. If $s>3 / 2$, there exists a unique $u \in C\left(\mathbb{R} ; H_{\mathrm{per}}^{s}([0, L])\right)$ such that $u$ solves the initial value problem

$$
\left\{\begin{array}{l}
u_{t}+2 u u_{x}-\left(\mathcal{M}_{\delta} u\right)_{x}=0, \quad(x, t) \in \mathbb{R} \times \mathbb{R} \\
u(0)=u_{0}
\end{array}\right.
$$

In addition, for all $T>0$ the data-solution map

$$
u_{0} \in H_{\mathrm{per}}^{s}([0, L]) \rightarrow u \in C\left([0, T] ; H_{\mathrm{per}}^{s}([0, L])\right),
$$

is continuous.

Proof. See Abdelouhab et al., [1].

Now, the ILW equation has the following three basic conserved quantities:

$$
E_{-1}(u)=\int_{0}^{L} u d x, \quad E_{0}(u)=\frac{1}{2} \int_{0}^{L} u^{2} d x
$$

and

$$
E_{1}(u)=\frac{1}{2} \int_{0}^{L}\left(\mathcal{M}_{\delta} u\right) u d x-\frac{1}{3} \int_{0}^{L} u^{3} d x .
$$

Indeed, from Theorem 6.2 and density arguments, we deduce that, for all $t$,

$$
E_{-1}(u(t))=E_{-1}\left(u_{0}\right), \quad E_{0}(u(t))=E_{0}\left(u_{0}\right), \quad \text { and } \quad E_{1}(u(t))=E_{1}\left(u_{0}\right) .
$$

Moreover, the ILW equation admits the following Hamiltonian structure:

$$
u_{t}=-2 u u_{x}+\left(\mathcal{M}_{\delta} u\right)_{x}=\partial_{x}\left(-u^{2}+\mathcal{M}_{\delta} u\right)=\partial_{x} E_{1}^{\prime}(u) .
$$

Our purpose is to describe Andrade and Pastor's approach in [7] for the case of equation (1.10) (we note that the strategy established in [7] is a generalization of the results in [28]). In fact, from Theorem 3.1 the wave speed $c$ given by $(1.12)$ depends smoothly on the elliptic modulus $k$ (see (3.11)). Our stability analysis will 
be based on this new parameter instead of the wave speed parameter $c$ (the analysis on the parameter $c$ is standard in the classical literature). Therefore, we need to establish a stability framework based on this new "wave speed" parameter $k$. Thus, by using [7] and [24], we consider, for every $k \in\left(0, k_{1}\right)$, the following smooth manifold in the space $\mathcal{W}$ :

(6.4) $\Sigma_{k}=\left\{u \in \mathcal{W} ; M_{k}(u)=M_{k}\left(\varphi_{k}\right)\right.$, where $\left.M_{k}(u):=\frac{d c}{d k} E_{0}(u)+\frac{d A}{d k} E_{-1}(u)\right\}$,

where $\varphi_{k}:=\varphi_{c(k)}$ and $A=A(k)=\frac{1}{L} \int_{0}^{L} \varphi_{k}^{2}(x) d x$. Now, the assumptions to obtain the orbital stability of $\varphi_{k}$ in the sense of Definition 6.1 are the following:

$\left(P_{0}\right)$ There exists a smooth curve of periodic solutions for (1.11) in the form,

$$
k \in J \subset \mathbb{R} \rightarrow \varphi_{k} \in H_{\text {per }}^{n}([0, L]) \cap H_{0}, \quad n \in \mathbb{N}
$$

$\left(P_{1}\right) \operatorname{ker}(\mathcal{L})=\left[\frac{d}{d x} \varphi_{k}\right]$

$\left(P_{2}\right) \mathcal{L}$ has a unique negative eigenvalue $\lambda$ which is simple;

$\left(P_{3}\right)\left\langle\mathcal{L}\left(\frac{\partial \varphi_{k}}{\partial k}\right),\left(\frac{\partial \varphi_{k}}{\partial k}\right)\right\rangle<0$.

Conditions $\left(P_{0}\right)-\left(P_{1}\right)-\left(P_{2}\right)$ have been established in Theorems 3.1 and 4.3 above. Concerning condition $\left(P_{3}\right)$, if we differentiate the equation in (1.11) with respect to $k$, we obtain the equality

$$
\mathcal{L}\left(\frac{\partial \varphi_{k}}{\partial k}\right)=-\frac{d c}{d k} \varphi_{k}-\frac{d A}{d k}=-M_{k}^{\prime}\left(\varphi_{k}\right)
$$

Thus, from Proposition 5.6, Remark 4.4 and the fact that $\varphi_{k} \in H_{0}$, we obtain, for every $k$ such that $c=c(k) \neq 0$,

$$
\left\langle\mathcal{L}\left(\frac{\partial \varphi_{k}}{\partial k}\right),\left(\frac{\partial \varphi_{k}}{\partial k}\right)\right\rangle=-\left\langle M_{k}^{\prime}\left(\varphi_{k}\right), \frac{\partial \varphi_{k}}{\partial k}\right\rangle=-\frac{1}{2} \frac{d c}{d k} \frac{d}{d k} \int_{0}^{L} \varphi_{k}^{2}(x) d x<0 .
$$

The main theorem of this section is now presented.

Theorem 6.3. Let $k \in\left(0, k_{1}\right)$ be fixed such that $c=c(k) \neq 0$. Then the periodic wave $\varphi_{k}=\varphi_{c(k)}$ in (1.12) is orbitally stable by the periodic flow of equation (1.10) in the sense of Definition 6.1.

For the reader's convenience, we shall give a sketch of the proof of Theorem 6.3. The proof of the next two lemmas follow from the ideas in [7], [8], [24], and [28].

Lemma 6.4. There exist $\varepsilon>0$ and a $C^{1}$-function, $\omega: U_{\varepsilon}\left(\varphi_{k}\right) \mapsto \mathbb{R}$, with

$$
U_{\varepsilon}\left(\varphi_{k}\right):=\left\{u \in \mathcal{W} ;\left\|u-\varphi_{k}\right\|_{\mathcal{W}}<\varepsilon\right\}
$$

such that

$$
\left\langle u(\cdot+\omega(u)), \frac{d}{d x} \varphi_{k}\right\rangle=0, \quad \text { for all } \quad u \in U_{\varepsilon}\left(\varphi_{k}\right)
$$


Lemma 6.5. Suppose that the conditions $\left(P_{0}\right),\left(P_{1}\right),\left(P_{2}\right)$, and $\left(P_{3}\right)$ are verified. Define the set

$$
\mathcal{A}_{k}:=\left\{\Phi \in \mathcal{W} ;\left\langle\Phi, M_{k}^{\prime}\left(\varphi_{k}\right)\right\rangle=\left\langle\Phi, \frac{d}{d x} \varphi_{k}\right\rangle=0\right\}
$$

Then, there exists a constant $C>0$ such that

$$
\langle\mathcal{L} \Phi, \Phi\rangle \geq C\|\Phi\|_{\mathcal{W}}^{2}, \quad \text { for all } \Phi \in \mathcal{A}_{k}
$$

Now, for $u \in \mathcal{W}$ we define the pseudo-metric

$$
\rho\left(u, \varphi_{k}\right):=\inf _{r \in[0, L]}\left\|u-\varphi_{k}(\cdot+r)\right\|_{\mathcal{W}}
$$

which indicates the distance between $u$ and the orbit generated by $\varphi_{k}$ via the translation symmetry, namely, $\Omega_{k}=\left\{\varphi_{k}(\cdot+r): r \in[0, L]\right\}$.

The following lemma establishes the existence of local minimizers on the manifold $\Sigma_{k}$.

Lemma 6.6. Suppose that the conditions $\left(P_{0}\right),\left(P_{1}\right),\left(P_{2}\right)$, and $\left(P_{3}\right)$ are verified. Define the functional

$$
\mathcal{F}_{k}=E_{1}+c E_{0}+A E_{-1}
$$

Then, there exist $\varepsilon>0$ and a constant $C(\varepsilon)>0$ satisfying

$$
\mathcal{F}_{k}(u)-\mathcal{F}_{k}\left(\varphi_{k}\right) \geq C(\varepsilon)\left[\rho\left(u, \varphi_{k}\right)\right]^{2}
$$

for all $u \in U_{\varepsilon}\left(\varphi_{k}\right) \cap \Sigma_{k}$.

Proof. Consider $u \in \mathcal{W}$. Since $\mathcal{F}_{k}$ is invariant under translations, one has $\mathcal{F}_{k}(u)=$ $\mathcal{F}_{k}(u(\cdot+r))$, for all $r \in \mathbb{R}$. Hence, it is suffices to show that

$$
\mathcal{F}_{k}(u(\cdot+\omega(u)))-\mathcal{F}_{k}\left(\varphi_{k}\right) \geq C\left[\rho\left(u, \varphi_{k}\right)\right]^{2},
$$

where $\omega$ is the smooth function obtained in Lemma 6.4. Thus, since $u \in \Sigma_{k}$, we deduce from Lemma 6.4 , the existence of a constant $C_{1} \in \mathbb{R}$ such that

$$
v:=u(\cdot+\omega(u))-\varphi_{k}=C_{1} M_{k}^{\prime}\left(\varphi_{k}\right)+y,
$$

where $y \in \mathcal{B}_{k}=\left[M_{k}^{\prime}\left(\varphi_{k}\right)\right]^{\perp} \cap\left[\frac{d}{d x} \varphi_{k}\right]^{\perp}$. Next, since $M_{k}$ is also invariant under translations, we can apply Taylor's formula to obtain

$$
M_{k}(u)=M_{k}(u(\cdot+\omega(u)))=M_{k}\left(\varphi_{k}\right)+\left\langle M_{k}^{\prime}\left(\varphi_{k}\right), v\right\rangle+\mathcal{O}\left(\|v\|_{\mathcal{W}}^{2}\right) .
$$

Hence, since $y \in \mathcal{B}_{k}$ one has $\left\langle M_{k}^{\prime}\left(\varphi_{k}\right), v\right\rangle=\left\langle M_{k}^{\prime}\left(\varphi_{k}\right), C_{1} M_{k}^{\prime}\left(\varphi_{k}\right)\right\rangle=C_{1} N$, where $N$ is a constant which is associated with the wave speed $c$. Thus, since $M_{k}(u)=M_{k}\left(\varphi_{k}\right)$, we obtain from (6.7) that

$$
C_{1}=\mathcal{O}\left(\|v\|_{\mathcal{W}}^{2}\right)
$$


Now, since $\mathcal{F}_{k}^{\prime}\left(\varphi_{k}\right)=0$ and $\mathcal{F}_{k}^{\prime \prime}\left(\varphi_{k}\right)=\mathcal{L}$, we can use Taylor's theorem to deduce

$$
\mathcal{F}_{k}(u)-\mathcal{F}_{k}\left(\varphi_{k}\right)=\frac{1}{2}\langle\mathcal{L} v, v\rangle+o\left(\|v\|_{\mathcal{W}}^{2}\right)
$$

Therefore, from (6.6) and (6.8), we have $\langle\mathcal{L} v, v\rangle=\langle\mathcal{L} y, y\rangle+\mathcal{O}\left(\|v\|_{\mathcal{W}}^{3}\right)$, that is, we conclude

$$
\mathcal{F}_{k}(u)-\mathcal{F}_{k}\left(\varphi_{k}\right)=\frac{1}{2}\langle\mathcal{L} y, y\rangle+o\left(\|v\|_{\mathcal{W}}^{2}\right)
$$

Since $y \in \mathcal{B}_{k}$, we obtain from Lemma 6.5 the existence of $C>0$ such that $\langle\mathcal{L} y, y\rangle \geq$ $C\|y\|_{\mathcal{W}}^{2}$. Thus,

$$
\mathcal{F}_{k}(u)-\mathcal{F}_{k}\left(\varphi_{k}\right) \geq \widetilde{C}\|y\|_{\mathcal{W}}^{2}+o\left(\|v\|_{\mathcal{W}}^{2}\right)
$$

where $\tilde{C}>0$. Therefore, from (6.6), we deduce that for $\varepsilon>0$ small enough, there exists $C=C(\varepsilon)>0$ such that

$$
\mathcal{F}_{k}(u)-\mathcal{F}_{k}\left(\varphi_{k}\right) \geq C\|v\|_{\mathcal{W}}^{2} \geq C\left[\rho\left(u, \varphi_{k}\right)\right]^{2} .
$$

This finishes the proof.

Proof of Theorem 6.3. The proof follows from Theorem 6.2, Lemma 6.6 and a convenient modification of Theorem 3.5 in [24] (see also [7]). We suppose that $\varphi_{k}$ is not orbitally stable and so we can select $w_{n}:=u_{n}(\cdot, 0) \in U_{1 / n}\left(\varphi_{k}\right) \cap H_{\text {per }}^{s}([0, L])$, $s>3 / 2$, and $\varepsilon>0$, such that $\left\|w_{n}-\varphi_{k}\right\|_{H_{\text {per }}^{s}} \rightarrow 0$, as $n \rightarrow \infty$, with

$$
\sup _{t \geq 0} \rho\left(u_{n}(\cdot, t), \varphi_{k}\right) \geq \varepsilon
$$

where $u_{n}(\cdot, t)$ is the corresponding solution of (6.2) with initial data $w_{n}$. Let us consider $\varepsilon>0$ satisfying Lemma 6.4. From continuity of $u_{n}(t)$ at $t \in \mathbb{R}$, we consider the smallest $t_{n}>0$ satisfying

$$
\rho\left(u_{n}\left(\cdot, t_{n}\right), \varphi_{k}\right)=\frac{\varepsilon}{2} .
$$

The following step in our analysis will be to determine the existence of $\alpha_{n}>0$ such that $\alpha_{n} u_{n}\left(\cdot, t_{n}\right) \in \Sigma_{k}$, for $n$ large. This is exactly the point in our approach where we will apply the strategy in [7]. Indeed, let us define $f_{n}: \mathbb{R} \rightarrow \mathbb{R}$, such that, for $n$ fixed,

$$
\begin{aligned}
f_{n}(\alpha) & =M_{k}\left(\alpha u_{n}\left(\cdot, t_{n}\right)\right)=\frac{\alpha^{2}}{2} \frac{d c}{d k} \int_{0}^{L}\left|u_{n}\left(\cdot, t_{n}\right)\right|^{2} d x+\alpha \frac{d A}{d k} \int_{0}^{L} u_{n}\left(\cdot, t_{n}\right) d x \\
& =: \alpha^{2} g_{n}+\alpha h_{n} .
\end{aligned}
$$

We note immediately that $f_{n}(0)=0, g_{n}>0$ and $M_{k}\left(\varphi_{k}\right)>0$. Thus, for all $n \in \mathbb{N}$ there exists $\alpha_{n}>0$ such that $f_{n}\left(\alpha_{n}\right)=M_{k}\left(\varphi_{k}\right)$. In other words, we guarantee the existence of $\left(\alpha_{n}\right)_{n \in \mathbb{N}} \subset \mathbb{R}$ satisfying

$$
M_{k}\left(\alpha_{n} u_{n}\left(\cdot, t_{n}\right)\right)=M_{k}\left(\varphi_{k}\right), \quad \text { for all } n \in \mathbb{N},
$$

that is, $\left(\alpha_{n} u_{n}\left(\cdot, t_{n}\right)\right)_{n \in \mathbb{N}} \subset \Sigma_{k}$. 
Next, let $\mathcal{T}_{k}(u):=\frac{d c}{d k} E_{0}(u)$ and $\mathcal{R}_{k}(u):=\frac{d A}{d k} E_{-1}(u)$. Then, since $E_{0}$ and $E_{-1}$ are continuous mappings, one has that $\mathcal{T}_{k}\left(w_{n}\right) \rightarrow \mathcal{T}_{k}\left(\varphi_{k}\right)=: g \neq 0, \mathcal{R}_{k}\left(w_{n}\right) \rightarrow$ $\mathcal{R}_{k}\left(\varphi_{k}\right)=: h$ and $M_{k}\left(w_{n}\right) \longrightarrow M_{k}\left(\varphi_{k}\right)$, as $n \rightarrow+\infty$. So,

$$
\begin{aligned}
\varrho_{n} & :=\left|\alpha_{n}^{2} \mathcal{T}_{k}\left(w_{n}\right)+\alpha_{n} \mathcal{R}_{k}\left(w_{n}\right)-\left(\mathcal{T}_{k}\left(w_{n}\right)+\mathcal{R}_{k}\left(w_{n}\right)\right)\right| \\
& =\left|M_{k}\left(\alpha_{n} u_{n}\left(\cdot, t_{n}\right)\right)-M_{k}\left(w_{n}\right)\right|=\left|M_{k}\left(w_{n}\right)-M_{k}\left(\varphi_{k}\right)\right| \longrightarrow 0,
\end{aligned}
$$

as $n \rightarrow+\infty$. On the other hand,

$0 \leq\left|\alpha_{n}^{2} \mathcal{T}_{k}\left(w_{n}\right)+\alpha_{n} \mathcal{R}_{k}\left(w_{n}\right)-(g+h)\right| \leq \varrho_{n}+\left|\mathcal{T}_{k}\left(w_{n}\right)-g\right|+\left|\mathcal{R}_{k}\left(w_{n}\right)-h\right| \longrightarrow 0$,

that is,

$$
z_{n}:=\alpha_{n}^{2} \mathcal{T}_{k}\left(w_{n}\right)+\alpha_{n} \mathcal{R}_{k}\left(w_{n}\right) \longrightarrow g+h .
$$

Therefore, statement (6.12) gives us that $\left(\alpha_{n}\right)_{n \in \mathbb{N}}$ is a bounded sequence and therefore, modulo a subsequence, one has $\alpha_{n} \longrightarrow \alpha_{0}$, as $n \rightarrow+\infty$. We will see that $\alpha_{0}=1$. Indeed, from (6.12) we get

$$
\left(1-\alpha_{0}\right)\left[\left(1+\alpha_{0}\right) g+h\right]=0 .
$$

Now, since

$$
1+\frac{h}{g}=1+\frac{\mathcal{R}_{k}\left(\varphi_{k}\right)}{\mathcal{T}_{k}\left(\varphi_{k}\right)}=1+\frac{1}{\mathcal{T}_{k}\left(\varphi_{k}\right)} \frac{d A}{d k} \int_{0}^{L} \varphi_{k}(\xi) d \xi=1+\frac{0}{\mathcal{T}_{k}\left(\varphi_{k}\right)}=1>0,
$$

we obtain that $\alpha_{0}>0$. Therefore, since $g \neq 0$, it follows from (6.13) that $\alpha_{0}=1$.

Next, we claim that

$$
\rho\left(u_{n}\left(\cdot, t_{n}\right), \alpha_{n} u_{n}\left(\cdot, t_{n}\right)\right) \longrightarrow 0, \quad n \rightarrow+\infty .
$$

In fact, since $\rho\left(u_{n}\left(\cdot, t_{n}\right), \varphi_{k}\right)=\varepsilon / 2$, there exist $r_{n} \in \mathbb{R}$ and $C_{2}>0$ such that

$$
\begin{aligned}
\left\|u_{n}\left(\cdot, t_{n}\right)\right\|_{\mathcal{W}} & \leq\left\|u_{n}\left(\cdot, t_{n}\right)-\varphi_{k}\left(\cdot+r_{n}\right)\right\|_{\mathcal{W}}+\left\|\varphi_{k}\left(\cdot+r_{n}\right)\right\|_{\mathcal{W}} \\
& <\varepsilon+\left\|\varphi_{k}\left(\cdot+r_{n}\right)\right\|_{\mathcal{W}}=C_{2},
\end{aligned}
$$

that is, $\left(\left\|u_{n}\left(\cdot, t_{n}\right)\right\|_{\mathcal{W}}\right)_{n \in \mathbb{N}}$ is a bounded sequence. Therefore, the convergence $\alpha_{n} \rightarrow 1$ and the relation

$$
\begin{aligned}
\rho\left(u_{n}\left(\cdot, t_{n}\right), \alpha_{n} u_{n}\left(\cdot, t_{n}\right)\right) & \leq\left\|u_{n}\left(\cdot, t_{n}\right)-\alpha_{n} u_{n}\left(\cdot, t_{n}\right)\right\|_{\mathcal{W}} \\
& \leq\left|1-\alpha_{n}\right| \cdot\left\|u_{n}\left(\cdot, t_{n}\right)\right\|_{\mathcal{W}},
\end{aligned}
$$

imply (6.14). Thus, an application of the triangle inequality and (6.10) show that $\left(\alpha_{n} u_{n}\left(\cdot, t_{n}\right)\right)_{n \in \mathbb{N}} \subset U_{\varepsilon}\left(\varphi_{k}\right)$. Hence, from Lemma 6.6 we conclude immediately the convergence

$$
\rho\left(\alpha_{n} u_{n}\left(\cdot, t_{n}\right), \varphi_{k}\right) \longrightarrow 0, \quad n \rightarrow+\infty .
$$

Finally, by using (6.14) and (6.16), we obtain that

$$
\frac{\varepsilon}{2}=\rho\left(u_{n}\left(\cdot, t_{n}\right), \varphi_{k}\right) \leq \rho\left(u_{n}\left(\cdot, t_{n}\right), \alpha_{n} u_{n}\left(\cdot, t_{n}\right)\right)+\rho\left(\alpha_{n} u_{n}\left(\cdot, t_{n}\right), \varphi_{k}\right) \longrightarrow 0,
$$

as $n \rightarrow+\infty$. Last fact gives us a contradiction, and the proof of Theorem 6.3 is completed. 
Remark 6.7. The positive and periodic wave $\phi_{\varsigma}$ in (4.18) is also orbitally stable by using a direct application of the arguments in [10].

Acknowledgements. The authors would like to thank the two anonymous referees for their valuable suggestions and constructive comments that allowed us to improve our manuscript.

\section{References}

[1] Abdelouhab, L., Bona, J. L., Felland, M. and Saut, J-C.: Nonlocal models for nonlinear dispersive waves. Phys. D 40 (1989), no. 3, 360-392.

[2] Ablowitz, M. J., Fokas, A.S., Satsuma, J. and Segur, H.: On the periodic intermediate long wave equation. J. Phys. A 15 (1982), no. 3, 781-786.

[3] Abramowitz, M. And Stegun, I. A.: Handbook of mathematical functions with formulas, graphs and mathematical tables. Dover Publications, New York, 1972.

[4] Albert, J. P.: Positivity properties and stability of solitary-wave solutions of model equations for long waves. Comm. Partial Differential Equations 17 (1992), no. 1-2, $1-22$.

[5] Albert, J. P. And Bona, J. L.: Total positivity and the stability of internal waves in fluids of finite depth. IMA J. Appl. Math. 46 (1991), no. 1-2, 1-19.

[6] Albert, J. P., Bona, J. L. And Henry, D. B.: Sufficient conditions for stability of solitary-wave equation of model equations for long waves. Phys. D 24 (1987), no. 1-3, 343-366.

[7] Andrade, T.P. And Pastor, A.: Orbital stability of periodic traveling-wave solutions for the regularized Schamel equation. Phys. D 317 (2016), 43-58.

[8] Angulo, J.: Nonlinear dispersive equations: existence and stability of solitary and periodic travelling wave solutions. Mathematical Surveys and Monographs 156, American Mathematical Society, Providence, 2009.

[9] Angulo, J., Bona, J. L. And Scialom, M.: Stability of cnoidal waves. Adv. Differential Equations 11 (2006), no. 12, 1321-1374.

[10] Angulo, J. And Natali, F.: Positivity properties of the Fourier transform and the stability of periodic travelling-wave solutions. SIAM J. Math. Anal. 40 (2008), no. 3, $1123-1151$.

[11] Angulo, J. And Natali, F.: Instability of periodic traveling waves for dispersive models. Differential Integral Equations 29 (2016), no. 9-10, 837-874.

[12] Benjamin, T. B.: The stability of solitary waves. Proc. Roy. Soc. (London) Ser. A 328 (1972), 153-183.

[13] Benjamin, T. B.: Lectures on nonlinear wave motion. In Nonlinear wave motion (Proc. AMS-SIAM Summer Sem., Clarkson Coll. Tech., Potsdam, NY, 1972), 3-47. Lecture in Appl. Math. 15, American Mathematical Society, Providence, RI, 1974.

[14] Bona, J. L.: On the stability theory of solitary waves. Proc Roy. Soc. London Ser. A 344 (1975), no. 1638, 363-374.

[15] Bona, J. L., Souganidis, P. E. And Strauss, W. A.: Stability and instability of solitary waves of Korteweg-de Vries type equations. Proc. Roy. Soc. London Ser. A 411 (1987), no. 1841, 395-412. 
[16] Bronski, J. C. And Johnson, M.: The modulational instability for a generalized Korteweg-de Vries equation. Arch. Ration. Mech. Anal. 197 (2010), no. 2, 357-400.

[17] Bronski, J. C., Johnson, M. And Kapitula, T.: An index theorem for the stability of periodic traveling waves of Korteweg-de Vries type. Proc. Roy. Soc. Edinburgh Sect. A 141 (2011), no. 6, 1141-1173.

[18] Bronski, J. C., Johnson, M. And Kapitula, T.: An instability index theory for quadratic pencils and applications. Comm. Math. Phys. 327 (2014), no. 2, 521-550.

[19] Bronski, J. C., Johnson, M. And Zumbrun, K.: On the modulation equations and stability of periodic generalized Korteweg-de Vries waves via Bloch decompositions. Phys. D 239 (2010), no. 23-24, 2057-2065.

[20] Byrd, P. F. And Friedman, M. D.: Handbook of elliptic integrals for engineers and scientists. Die Grundlehren der mathematischen Wissenschaften 67, Springer-Verlag, New York-Heidelberg, 1971.

[21] Deconinck, B. And Kapitula, T.: The orbital stability of the cnoidal waves of the Korteweg-de Vries equation. Phys. Lett. A 374 (2010), no. 39, 4018-4022.

[22] Deconinck, B. And Kapitula, T.: On the spectral and orbital stability of spatially periodic stationary solutions of generalized Korteweg-de Vries equations. In Hamiltonian partial differential equations and applications, 285-322. Fields Inst. Commun. 75, Fields Inst. Res. Math. Sci., Toronto, ON, 2015.

[23] Deconinck, B. And Nivala, M.: The stability analysis of the periodic traveling wave solutions of the mKdV equation. Stud. Appl. Math. 126 (2011), no. 1, 17-48.

[24] Grillakis, M., Shatah, J. and Strauss, W.: Stability theory of solitary waves in the presence of symmetry I. J. Funct. Anal. 74 (1987), no. 1, 160-197.

[25] Grillakis, M., Shatah, J. and Strauss, W.: Stability theory of solitary waves in the presence of symmetry II. J. Funct. Anal. 94 (1990), no. 2, 308-348.

[26] Haragus, M. And Kapitula, T.: On the spectra of periodic waves for infinitedimensional Hamiltonian systems. Phys. D 237 (2008), no. 20, 2649-2671.

[27] Hur, V. And Johnson, M.: Stability of periodic traveling waves for nonlinear dispersive equations. SIAM J. Math. Anal. 47 (2015), no. 5, 3528-3554.

[28] Johnson, M.: Nonlinear stability of periodic traveling wave solutions of the generalized Korteweg-de Vries equation. SIAM J. Math. Anal. 41 (2009), no. 5, 1921-1947.

[29] Johnson, M.: On the stability of periodic solutions of the generalized BenjaminBona-Mahony equation. Phys. D 239 (2010), no. 19, 1892-1908.

[30] Johnson, M.: Stability of small periodic waves in fractional KdV-type equations. SIAM J. Math. Anal. 45 (2013), no. 5, 3168-3193.

[31] Kapitula, T. And Promislow, K.: Spectral and dynamical stability of nonlinear waves. Applied Mathematical Sciences 185, Springer, New York, 2013.

[32] Kapitula, T. and Stefanov, A.: A Hamiltonian-Krein (instability) index theory for for solitary waves to the KdV-like eigenvalue problems. Stud. Appl. Math. 132 (2014), no. 3, 183-211.

[33] Karlin, S.: Total positivity. Stanford University Press, Stanford, 1968.

[34] Lin, Z.: Instability of nonlinear dispersive solitary waves. J. Funct. Anal. 255 (2008), no. 5, 1091-1124.

[35] Lopes, O.: A linearized instability result for solitary waves. Discrete Contin. Dyn. Syst. 8 (2002), no. 1, 115-119. 
[36] Nakamura, A. And Matsuno, Y.: Exact one-and two-periodic wave solutions of fluids of finite depth. J. Phys. Soc. Jpn. 48 (1980), 653-657.

[37] PARKer, A.: Periodic solutions of the intermediate long-wave equation: a nonlinear superposition principle. J. Phys. A 25 (1992), no. 7, 2005-2032.

[38] Weinstein, M. I.: Lyapunov stability of ground states of nonlinear dispersive evolution equations. Comm. Pure Appl. Math. 39 (1986), no. 1, 51-68.

[39] Weinstein, M. I.: On the structure and formation of singularities in solutions to nonlinear dispersive evolution equations. Commun. Partial Differential Equations 11 (1986), no. 5, 545-565.

[40] Weinstein, M. I.: Nonlinear Schrödinger equation and sharp interpolation estimates. Commun. Math. Phys. 87 (1982/83), no. 4, 567-576

Received April 7, 2015; revised January 1, 2016.

Jaime Angulo Pava: Institute of Mathematics and Statistics, State University of São Paulo, São Paulo, SP, Brazil.

E-mail: angulo@ime.usp.br

Eleomar Cardoso Jr.: Federal University of Santa Catarina, Blumenau, SC, Brazil.

E-mail: eleomar.jr@hotmail.com

FÁBio Natali: Department of Mathematics, State University of Maringá, Maringá, PR, Brazil.

E-mail: fmanatali@uem.br

The research of J. Angulo and F. Natali was partially supported by Grant CNPq/Brazil. E. Cardoso Jr. was supported by CAPES/Brazil. 